\title{
TUBB1 mutations cause thyroid dysgenesis associated with abnormal platelet physiology
}

\author{
Athanasia Stoupa ${ }^{1,2,3,4}$, Frédéric Adam ${ }^{5}$, Dulanjalee Kariyawasam ${ }^{3,4}$, Catherine Strassel $^{6}$, \\ Sanjay Gawade7, Gabor Szinnai ${ }^{7,8}$, Alexandre Kauskot ${ }^{5}$, Dominique Lasne ${ }^{5,9}$, Carsten Janke ${ }^{10,11}$ (iD), \\ Kathiresan Natarajan ${ }^{10,11, \dagger}$, Alain Schmitt ${ }^{1}$, Christine Bole-Feysot ${ }^{12}$, Patrick Nitschke ${ }^{13}$, \\ Juliane Léger ${ }^{3,14,15,16}$, Fabienne Jabot-Hanin ${ }^{13}$, Frédéric Tores ${ }^{13}$, Anita Michel ${ }^{6}$, Arnold Munnich ${ }^{17,18}$, \\ Claude Besmond $^{17}$, Raphaël Scharfmann ${ }^{1}$, François Lanza ${ }^{6}$, Delphine Borgel ${ }^{5,9}$, Michel Polak ${ }^{1,2,3,4,19}$ \& \\ Aurore Carré ${ }^{1,2,3, *}$ iD
}

\begin{abstract}
The genetic causes of congenital hypothyroidism due to thyroid dysgenesis (TD) remain largely unknown. We identified three novel TUBB1 gene mutations that co-segregated with TD in three distinct families leading to $1.1 \%$ of TUBB1 mutations in TD study cohort. TUBB1 (Tubulin, Beta 1 Class VI) encodes for a member of the $\boldsymbol{\beta}$ tubulin protein family. TUBB1 gene is expressed in the developing and adult thyroid in humans and mice. All three TUBBI mutations lead to non-functional $\alpha / \beta$-tubulin dimers that cannot be incorporated into microtubules. In mice, Tubb1 knock-out disrupted microtubule integrity by preventing $\beta 1$-tubulin incorporation and impaired thyroid migration and thyroid hormone secretion. In addition, TUBB1 mutations caused the formation of macroplatelets and hyperaggregation of human platelets after stimulation by low doses of agonists. Our data highlight unexpected roles for $\beta 1$ tubulin in thyroid development and in platelet physiology. Finally, these findings expand the spectrum of the rare paediatric diseases related to mutations in tubulin-coding genes and provide new insights into the genetic background and mechanisms involved in congenital hypothyroidism and thyroid dysgenesis.
\end{abstract}

Keywords congenital hypothyroidism; macroplatelets; mutations; thyroid dysgenesis; TUBB1

Subject Categories Genetics, Gene Therapy \& Genetic Disease; Haematology DOI 10.15252/emmm.201809569 | Received 19 July 2018 | Revised 18 October 2018 | Accepted 19 October 2018| Published online 16 November 2018

EMBO Mol Med (2018) 10: e9569

\section{Introduction}

Thyroid dysgenesis (TD) is a feature in $65 \%$ of patients with congenital hypothyroidism $(\mathrm{CH})$, the most common neonatal endocrine disorder affecting one in 2,500-3,500 newborns (Deladoëy et al, 2011; Barry et al, 2016). In France, the prevalence of $\mathrm{CH}$ due to TD is estimated in 1/5,000 (Barry et al, 2016). TD includes a vast spectrum of developmental thyroid anomalies encompassing athyreosis, thyroid ectopia, hypoplasia of an orthotopic gland, and hemithyroid (Barry et al, 2016; Stoupa et al, 2016). During embryogenesis, the midline thyroid anlage appears on embryonic day E.8.5 in mice and at 3 gestational weeks (GW) in humans. The midline anlage and ultimobranchial bodies migrate and fuse in the definitive

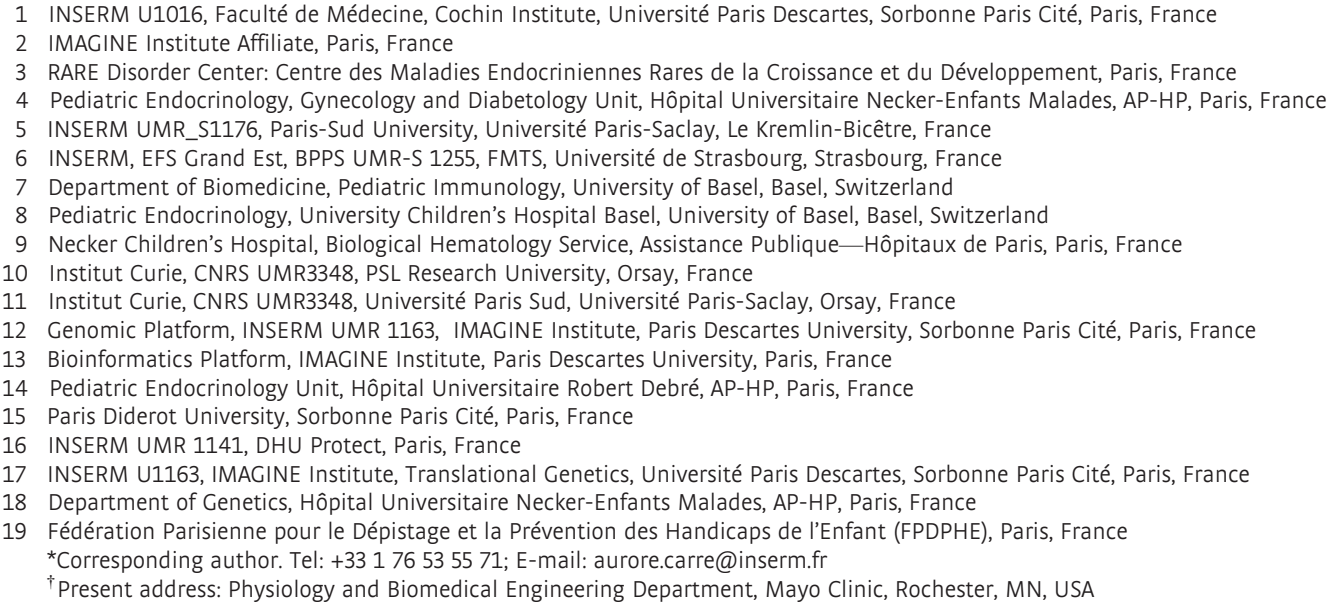


pretracheal position on E13.5 in mice and at $7 \mathrm{GW}$ in humans (Trueba et al, 2005; Nilsson \& Fagman, 2013). The cells differentiate into thyrocytes organized in follicles or C cells (Appendix Table S1; Szinnai et al, 2007). Abnormalities at any step of thyroid development may result in TD associated with hypothyroidism or not (Maiorana et al, 2003). Previous studies of sporadic and familial TD covering a wide clinical spectrum identified mutations in nine genes: PAX8, NKX2-1, FOXE1, NKX2-5, TSHR, GLIS3, NTN1, JAG1 and BOREALIN (Dentice et al, 2006; Senée et al, 2006; Carré et al, 2009, 2014, 2017; Sura-Trueba et al, 2009; Ramos et al, 2014; Opitz et al, 2015; de Filippis et al, 2016). However, mutations in these genes are found in only $5 \%$ of all patients with TD and identification of causative mutations remains a challenging task. Our objectives were to extend the knowledge on the genetic basis of $\mathrm{CH}$ and TD. We performed WES (whole exome sequencing; Choi et al, 2009; Hildebrandt et al, 2009) for siblings with $\mathrm{CH}$ and thereby identified a mutation in TUBB1 gene. Then, we analysed 270 TD cases by targeted NGS including the TUBB1 gene. We identified two more TUBB1 mutations in patients with $\mathrm{CH}$ and $\mathrm{TD}$.

TUBB1 (Tubulin, Beta 1 Class VI) encodes for a member of the $\beta$ tubulin protein family. $\beta$-tubulins are one of two core protein families that heterodimerize to form $\alpha / \beta$-tubulin dimers, which assemble into microtubules, one of the major cytoskeletal structures. The $\beta 1$ isotype of tubulin (TUBB1) has been described as specifically expressed in platelets and megakaryocytes and involved in proplatelet formation and platelet release (Patel et al, 2015). Few mutations of TUBB1 have been identified in patients with a rare autosomal dominant disease congenital macrothrombocytopaenia, in which impaired microtubule assembly results in low platelet counts and macroplatelets (Kunishima et al, 2009; 2014; Bastida et al, 2018; Johnson et al, 2016; Burley et al, 2018). Tubb1-knockout mice have thrombocytopaenia and spherical platelets (Schwer et al, 2001), but not known thyroid abnormalities. Our results highlight a hitherto unsuspected role for a specific tubulin isotype, Tubb1, in thyroid development and disease and extend our knowledge on genetic background of $\mathrm{CH}$.

\section{Results}

\section{Identification of TUBB1 mutations in a family with TD}

Family F1 is a consanguineous family of Algerian descent. The parents are first cousins (I.1, I.2) with five children including two females [II.1 (patient P1) and II.2 (patient P2)] with CH. Both patients were born at full term and diagnosed with $\mathrm{CH}$ by routine neonatal screening (Fig 1), which showed thyroid-stimulating hormone (TSH) elevation (164 and $177 \mu \mathrm{IU} / \mathrm{ml}$ in $\mathrm{P} 1$ and $\mathrm{P} 2$, respectively). On days 13 and 11, TSH was 67 and $202 \mu \mathrm{IU} / \mathrm{ml}$ in P1 and $\mathrm{P} 2$, respectively (normal range, $\mathrm{N}: 0.3-7 \mu \mathrm{IU} / \mathrm{ml}$ ), and free thyroxine (T4) was 14 and $13.3 \mathrm{pmol}$ in $\mathrm{P} 1$ and $\mathrm{P} 2$, respectively ( $\mathrm{N}$ : 9.5-25 pmol; Fig 1). L-thyroxine therapy was initiated. ${ }^{123}$ I scintigraphy showed thyroid ectopia in both siblings. Another sibling (II.5, P3), aged 12 years, had thyroid hypoplasia (thyroid volume, $3.1 \mathrm{ml}$; $\mathrm{N}: 7 \pm 3 \mathrm{ml})$ with a small right pyramidal lobe $(17 * 2 \mathrm{~mm})$ and normal thyroid function tests. The parents had normal thyroid function, and two other siblings (II.3 and II.4) had normal thyroid function but were not able to undergo thyroid ultrasonography.
To look for genetic causes of $\mathrm{CH}$ in $\mathrm{P} 1$ and $\mathrm{P} 2$, we performed whole exome sequencing (WES) using the variant filtering and prioritization strategy described in Appendix Fig S1. Using the recessive transmission model, WES identified a novel missense homozygous TUBB1 mutation (c.479C>T, p.P160L, rs759117911) in both siblings with $\mathrm{CH}$ (P1 and $\mathrm{P} 2$ ) and in the sibling with thyroid hypoplasia (P3; Fig 1). Both parents and sibling II.3 were carriers. The remaining sibling (II.4) did not carry the mutation. WES identified no variants in genes known to be associated with TD or thyroid dyshormonogenesis.

\section{Search for TUBB1 mutations in a cohort with thyroid dysgenesis (TD) and congenital hypothyroidism (CH)}

After identification of the above-described novel TUBB1 mutation, we used targeted next-generation sequencing (NGS) to assess TUBB1 in a cohort of 270 patients with $\mathrm{CH}$ and TD. In a second family (F2) with a father (I.2) of Moroccan and a mother (I.1) of French descent, a female with $\mathrm{CH}$ and thyroid gland ectopia (P4, II.1) had a heterozygous TUBB1 mutation (c.318C $>$ G, p.Y106X; Fig 1). $\mathrm{CH}$ was diagnosed upon routine neonatal screening (TSH, $250 \mu \mathrm{IU} / \mathrm{ml}$ ) and confirmed on day 15 (TSH, 1,100 $\mu \mathrm{IU} / \mathrm{ml}$; free $\mathrm{T} 4$, $3.5 \mathrm{pmol} / \mathrm{l}$; and free T3, $2.45 \mathrm{pmol} / \mathrm{l}$ ). Thyroid scintigraphy showed an ectopic thyroid. The father carried the same heterozygous mutation; unfortunately, thyroid ultrasound was not performed, and complete phenotype was therefore not possible. In a paternal aunt (I.3, P5), an evaluation at 26 years of age for obesity and depression showed mild hypothyroidism (TSH, $6.6 \mu \mathrm{IU} / \mathrm{ml}$; N: 0.1-5.5 $\mu \mathrm{IU} / \mathrm{ml}$; free T4, $8.7 \mathrm{pmol} / \mathrm{l}$; N: 9.8-23.1 pmol/l). Thyroid ultrasonography and scintigraphy showed right hemithyroid.

In a third family (F3), a patient (II.1, P6) with $\mathrm{CH}$ and an ectopic thyroid was shown by targeted NGS to have a heterozygous frameshift TUBB1 mutation (c.35delG, p.Cys12Leufs*12, rs77324804) that created a premature stop codon at amino acid 23 (Fig 1). $\mathrm{CH}$ was diagnosed neonatally based on TSH elevation $(476 \mu \mathrm{IU} / \mathrm{ml})$ and low free T4 and T3 levels ( 8 and $5.6 \mathrm{pmol} / \mathrm{l}$, respectively). L-thyroxine therapy was started at 11 days of age. Both parents were of French descent. The father (I.1, P7) carried the same heterozygous mutation and had normal thyroid function with mild thyroid lobe asymmetry by ultrasonography (right lobe, $6.9 \mathrm{ml}$; left lobe, $5 \mathrm{ml}$ ). The other siblings and mother had normal thyroid function and morphology and did not carry the mutation.

By targeted NGS, neither P4 nor P6 had any variants in genes known to cause $\mathrm{CH}$ (with TD or dyshormonogenesis).

In the Exome Aggregation Consortium (ExAC) database, estimated allele frequencies are 0.000008 for the c.479C $>\mathrm{T}$ mutation (rs759117911, 20:57598961 C/T) and 0.000025 for the c35delG (rs773248042, 20:57594611 TG/T) mutation. Neither mutation has been reported in homozygous form. The c. $318 \mathrm{C}>\mathrm{G}$ variation has not been reported in public databases. The in silico prediction tools, PolyPhen-2, SIFT, predict that c.479C $>\mathrm{T}$ is probably damaging and deleterious, respectively, with a PHRED-scaled CADD score of 32 (damaging: > 15; Kircher et al, 2014). The other two mutations create a premature stop codon and have PHRED-scaled CADD score of 35. CADD is a prediction algorithm, which integrates information contained in more than 60 diverse annotations of genetic variation into a single score. The higher the CADD score, the higher the deleteriousness probability of the variant investigated. 
F1, c479C>T, p.P160L

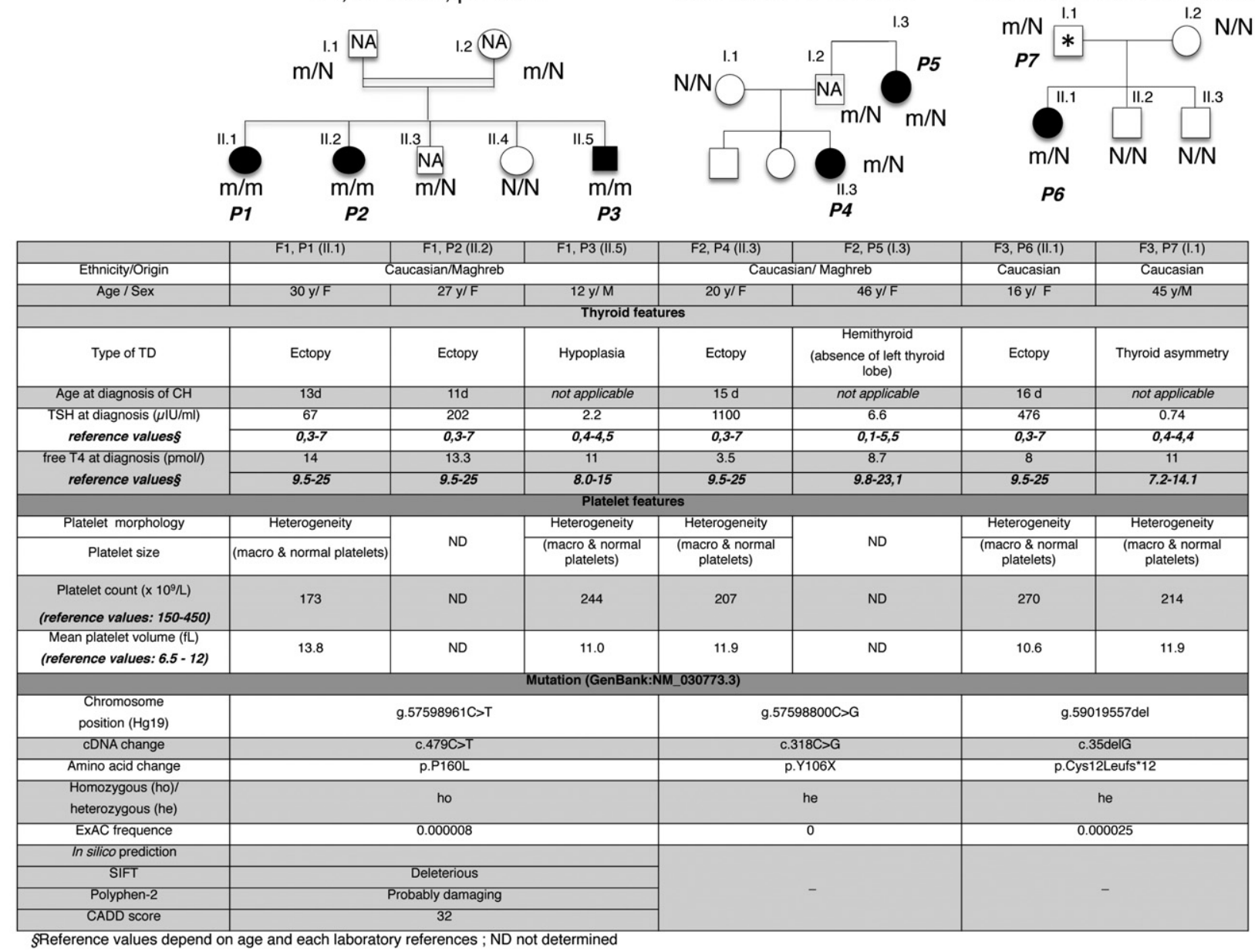

Figure 1. Pedigrees and clinical table.

Pedigrees of three families with TUBB1 mutations. Family F1 has three affected individuals with homozygous mutations, family F2 has two affected individuals with heterozygous mutations, and family F3 has two affected individuals with heterozygous mutations. Thus, all seven patients (P1-P7) carry at least one mutated allele and have thyroid dysgenesis (TD) and macroplatelets. The patients are represented with filled symbols and unaffected family members with open symbols. NA, not available thyroid ultrasonography; *mild thyroid asymmetry (possibly normal) with normal thyroid function; N, not mutated; m, mutated.

A Burden test was applied to determine whether TUBB1 was significantly enriched in rare variants in the 270 patients with $\mathrm{CH}$ and TD versus 406 Caucasian controls from the 1000 Genomes project. The patients in the TD cohort had athyreosis, ectopia, hemithyroid or hypoplasia. In the TD group, 14/270 patients (5.2\%) exhibited at least one rare functional variant in TUBB1 compared with $8 / 406$ controls ( $2 \%$ ). This difference is significant from a statistical point of view $(P=0.0227)$. None of the patients in our cohort with TUBB1 mutations had athyreosis. Performing the same test after excluding the 77 patients with athyreosis increased the significance of the difference of TD group $(n=193)$ versus controls $(P=0.0095)$. The list of rare functional variants found in TUBB1 is provided in Table EV1.

The three amino acids affected by the TUBB1 mutations are strictly conserved across species, from humans to zebrafish, and across all $\beta$-tubulins (Fig 2A). All three mutations were located in the first part of TUBB1, i.e., in the N-terminal domain needed for guanosine triphosphate (GTP) activity (Fig 2B). The c.318C $>\mathrm{G}$ and
c.35delG mutations created a premature stop codon, thereby removing the intermediate and C-terminal domains required for microtubule-associated protein (MAP) binding (Nogales et al, 1998).

In sum, we identified three TUBB1 mutations in three independent families of patients with $\mathrm{CH}$ and $\mathrm{TD}$ chiefly manifesting as thyroid gland ectopia. Thus, we found $1.1 \%$ of TUBB1 mutations in patients affected with $\mathrm{CH}$ and $\mathrm{TD}$ in our cohort.

\section{B1-tubulin is expressed in the developing thyroid in humans and mice}

$\beta 1$-tubulin expression has so far been reported only in megakaryocytes and platelets (Wang et al, 1986; Lecine et al, 2000). Our finding of TUBB1 mutations in patients with TD prompted us to look for $\beta 1$-tubulin expression in thyroid tissue. In human thyroid tissue, TUBB1 mRNA was expressed at 8,10 and $12 \mathrm{GW}$ and in adulthood (Fig 3A). In mouse thyroid tissue, Tubb1 was expressed at E13.5 and strongly at E15.5, E17.5 and adulthood (Fig 3B). To refine our 
A

$\stackrel{100}{\stackrel{125}{\perp}} \stackrel{150}{\mid}$

TUBB1 Homo sapiens Y106X NNWAKGHXTEGAELIENVLEVVRHESESCDCLOGFOIVHSLGGGTGSGMGTLLMNKIREEYPDRIM
TUBB1 Homo sapiens P160L NNWAKGHYTEGAELIENVLEVVRHESESCDCLOGFIVHSLGGGTGSGMGTLLMNKIREEYLDRIM TUBB1 Homo sapiens P160L NNWAKGHY YEGAELIIENVLEVVRHESESCDCLQGFQIVHSLGGGTGSGMGTLLMNKIREEYLDRIM TUBB1 Pan troglodytes NNWAKGHY YEGAELIENVLEVVRHESESCDCLOGFOIVHSLGGGTGSGMGTLLMNKIREEYPDRIM Tubb1 Mus musculus NNWAKGHY YEEGAELIENVMDVVRRESESCDCLQGFQIVHSLGGGTGSGMGTLLMNKIREEYPDRIL Tubb1 Rat norvegicus NNWAKGHYTEGAELIETVMDVVRRESESCDCLQGFQIVHSLGGGTGSGMGTLLMNKIREEYPDRIL TUBB1 Bos taurus NNWAKGYY YTEGAELVDRVLDAVRTEAEGCDCLQGFQLVHSLGGGTGSGMGTLLIGKIREEYPDRIL TUBB1 Sus scrofa NNWAKGHYTEGAELVEGVLDAVRSEAEGCDCLQGFOVVHSLGGGTGSGMGTLLMGKIREEFPDRIL Tubb1 Danio renio

TUBB1 Homo sapiens TUBB2A Homo sapiens TUBB2B Homo sapiens TUBB3 Homo sapiens TUBB4A Homo sapiens TUBB4B Homo sapiens TUBB5 Homo sapiens TUBB6 Homo sapiens TUBB8 Homo sapiens

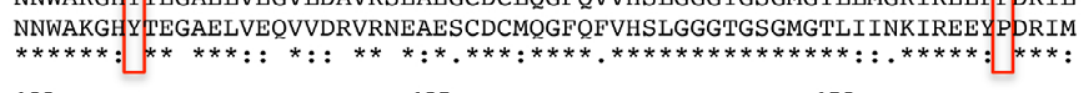

$\stackrel{125}{100} \stackrel{150}{1}$ NNWAKGHYIEGAELVDSVLDVVRKESESCDCLQGFQLTHSLGGGTGSGMGTLLISKIREEY PDRIM NNWAKGHY TEGAELVDSVLDVVRKESESCDCLOGFOLTHSLGGGTGSGMGTLLISKIREEYPDRIM NNWAKGH Y TEGAELVDSVLDVVRKECENCDCLQGFQLTHSLGGGTGSGMGTLLISKVREEY PDRIM NNWAKGHY YEGAELVDAVLDVVRKEAESCDCLQGFQLTHSLGGGTGSGMGTLLISKIREEF PDRIM NNWAKGH Y YEGAELVDSVLDVVRKEAESCDCLQGFQLTHSLGGGTGSGMGTLLISKIREEYPDRIM NNWAKGH Y TEGAELVDSVLDVVRKEAESCDCLQGFQLTHSLGGGTGSGMGTLLISKIREEY PDRIM NNWAKGH Y TEGAELVDAVLDVVRKECEHCDCLQGFOLTHSLGGGTGSGMGTLLISKIREEF PDRIM NNWAKGHYIEGAELMESVMDVVRKEAESCDCLQGFQLTHSLGGGTGSGMGTLLLSKIREEYPDRI I

\section{B

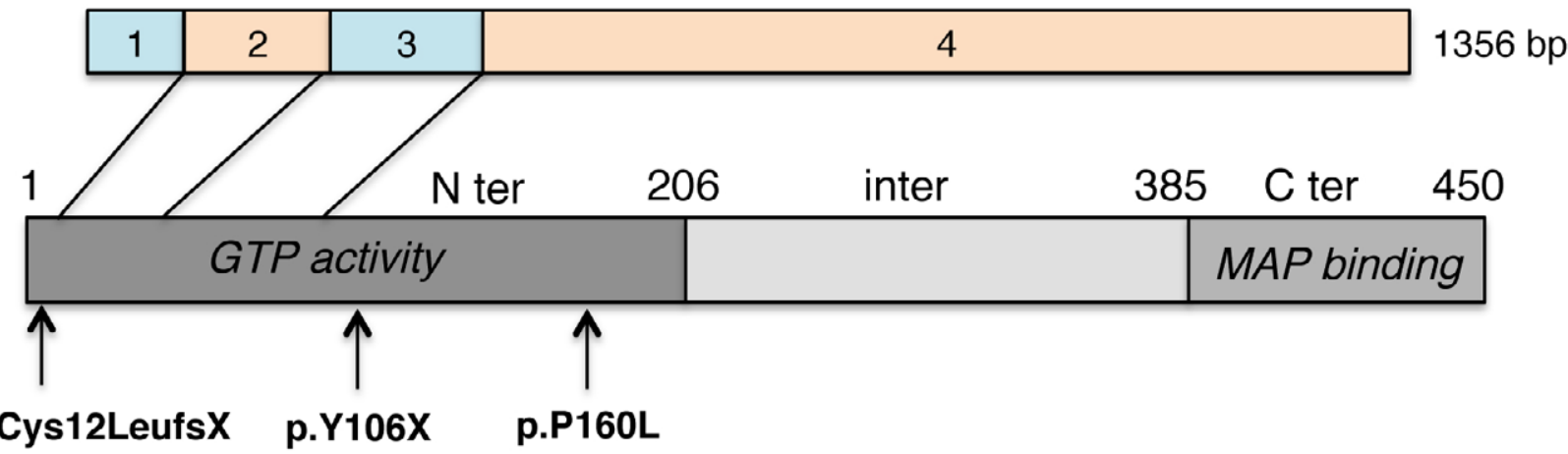 \\ pCys12LeufsX p.Y106X p.P160L \\ p.T107P \\ p.R241W p.F260S p.R359W p.R242L p.R318W \\ p.Q423X}

Figure 2. Molecular genetics.

A Alignment and conservation of residues encoded by TUBB1 orthologues and genes encoding for other $\beta$-tubulins (TUBB2A, TUBB2B, TUBB3, TUBB4A, TUBB4B, TUBB5, TUBB6 and TUBB8). Mutations are shown in green. Mutated aminoacids through species or through all TUBB are shown in red boxes.

B Location of TUBBI mutations in the CDNA and of the corresponding changes in the protein. Exons are represented by boxes numbered from 1 to 4 . The dark grey box represents the protein domain responsible for encoding guanosine triphosphate (GTP) and the light grey box the domain for microtubule-associated protein (MAP) binding. The arrows show the consequences of the three TUBBI mutations in our patients, all of which are in the GTP domain. Mutations of this study are in bold, and published mutations associated with congenital macrothrombocytopaenia are in italic.

study of Tubb1 expression, we used cells sorted from mice thyroid tissue based on well-accepted markers (Gawade et al, 2016) with stringent sorting regions, including the brightest cells for each selected marker (Pecam for endothelial cells, EpCAM for epithelial cells, Pdgfra for fibroblasts and CD45 for leucocytes). As expected, expression was strongest in platelets sorted using the specific megakaryocyte lineage marker CD41 (Appendix Fig S2A). However, Tubb1 was also expressed in EpCAM-positive epithelial-cell populations containing thyrocytes, at E17.5 and in adulthood (Fig 3C).

Similarly, in human thyroid tissue, immunohistochemistry showed $\beta 1$-tubulin expression in the cytoplasm of thyroglobulin (TG)-producing thyrocytes at $12 \mathrm{GW}$ (Fig 3D). Comparable findings were obtained with mouse thyroid tissue (Appendix Fig S2B). No staining was observed in the thyroid tissue of $\mathrm{Tubb}^{-/-}$mice
(Appendix Fig S2C). These data established that $\beta 1$-tubulin is expressed in thyrocytes.

\section{Functional in vitro analysis of disease-causing mutations}

To further investigate the implication of TUBB1 gene mutations in thyroid disease, we transfected the mutations into the Nthy-ori 3-1 cell line. Only the c479C > T (p.P160L) mutation could be studied in this model. The c318C >G (p.Y106X) and c.35delG (p.Cys12Leufs*12) mutations created premature stop codons that yielded truncated proteins (Appendix Fig S3) that cannot form functional $\alpha / \beta$-tubulin dimers and thus cannot get incorporated into microtubules (Nogales et al, 1998; Joe et al, 2009). The pathogenic role of these mutations is thus loss-of-function. 
A

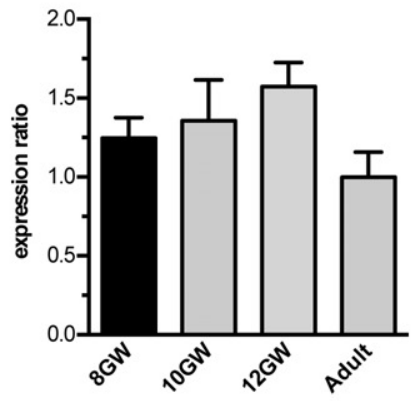

B

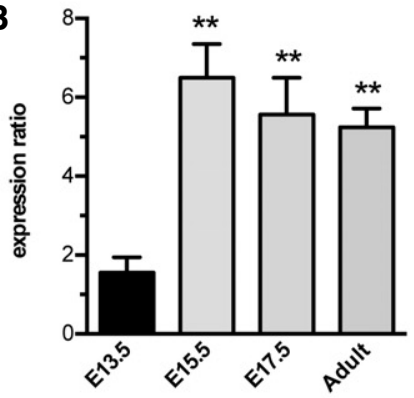

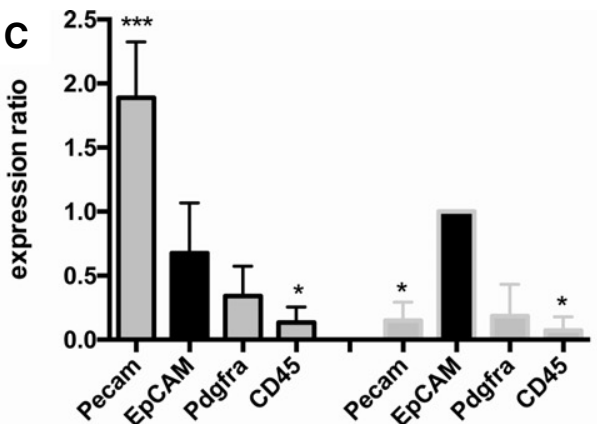

E17.5
$\mathbf{F}$

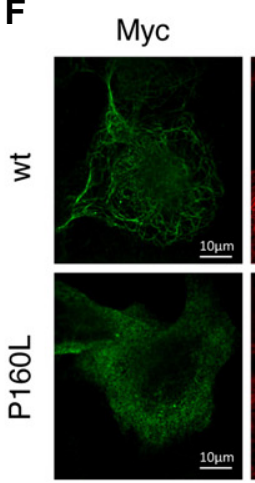

Adult

E

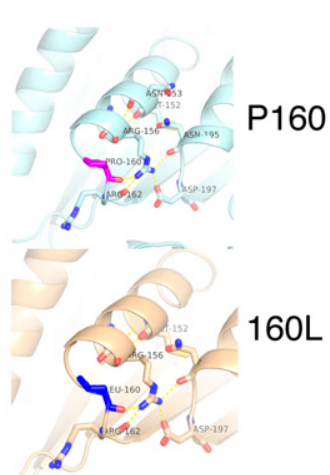

TUBB1/TG
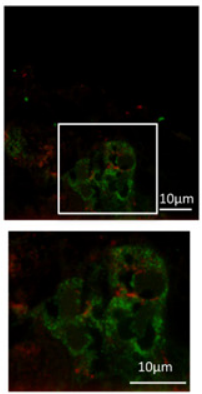
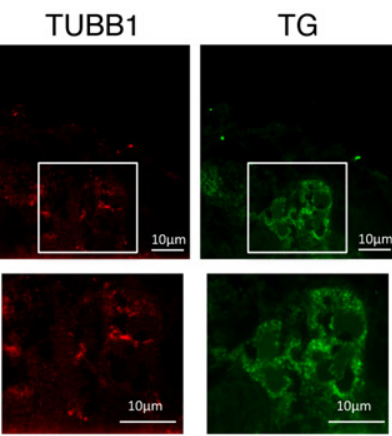

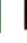

Figure 3. $\beta 1$-tubulin expression in the developing thyroid and deleterious effect of TUBB1 mutations.

A TUBB1 is expressed in the developing human thyroid at 8 gestational weeks (CW), $10 \mathrm{GW}$ and $12 \mathrm{GW}$ and in the adult human thyroid: quantitative PCR results normalized to one of three thyroid tissues at $8 \mathrm{GW}$ value (in black) and peptidylprolyl isomerase A. Experiments with four tissues per stage. Results are reported as mean \pm SEM. Statistical comparisons versus $8 \mathrm{CW}$ (in black) using ANOVA test showed no significant differences.

B Tubb1 is expressed in the developing mouse thyroid at E13.5, E15.5 and E17.5 and in the adult mouse thyroid: quantitative PCR results normalized to one of three thyroid tissues at E13.5 value and peptidylprolyl isomerase A. Experiments with four tissues per stage. Results are reported as mean \pm SEM. Statistical comparisons versus E13.5 (black), ${ }^{*} P<0.01$ using ANOVA test.

C Sorted mouse thyroid cells, Tubb1 expression in endothelial cells (Pecam-positive cells), epithelial cells (EPCAM), fibroblasts (Pdgfra) and leucocytes (CD45) at E17.5 and adulthood: quantitative PCR results (normalized to EPCAM-positive cells at adult stage and peptidylprolyl isomerase A). Mean \pm SEM of three independent cellsorting experiments. Statistical comparisons versus epithelial cells (in black) at each stage, ${ }^{\star} P<0.05$, ${ }^{* \star *} P<0.001$ using ANOVA test.

D Immunohistofluorescence of $\beta 1$-tubulin (in red), thyroglobulin (TG, in green) and both merged (from left to right) at $12 \mathrm{GW}$ in human thyroid tissue. The boxes delineate the enlarged areas shown below.

E Structural representation of wild-type and p.P160L mutated $\beta 1$-tubulin showing the change in conformation surrounding helix H4. Top: proline at position 160 in pink; bottom: substituting leucine at the same position in blue.

F Localization of transfected Myc-tagged wild-type and mutant (P160L) $\beta 1$-tubulin in Nthy cells. Immunostaining with anti-Myc antibody (in green) and microtubule cytoskeleton stained with $\alpha$-tubulin antibody (in red). Note that $\beta 1$-tubulin -P160L is found throughout the cytoplasm showing puncta appearance, whereas wildtype $\beta 1$-tubulin colocalizes with the microtubules.

\section{Structural modelling of p.P160L mutant $\beta 1$-tubulin}

To assess the consequences of the P160L mutation on $\beta 1$-tubulin function, the sole missense mutation that should give rise to a fulllength protein, we first compared the configurations of the mutated and wild-type proteins. The P160L mutation is located at the end of helix H4 (Fig 3E). In wild-type $\beta 1$-tubulin, the proline residue stabilizes loop H4-S5 and places R156 in a position that promotes a salt bridge interaction with D197 in the $\beta$-sheet S6. Furthermore, R162 in loop H4-S5 and N195 in the $\beta$-sheet S6 establish hydrogen bonds with R156. Mutation of proline 160 to leucine might affect the loop conformation of the H4-S5 loop and thus disrupting the interaction network mediated by R156. Hence, the P160L mutation is most likely affecting the conformation of the $\beta$-tubulin, which could lead to dysfunctions of the $\alpha / \beta$-tubulin dimer.
In vitro consequences of the p.P160L $\beta 1$-tubulin mutation on microtubule incorporation

Following the predictions from the modelling, we examined the ability of $\beta 1$-tubulin P160L protein to incorporate within the microtubule network in the Nthy cell line. We used a vector containing human TUBB1 cDNA tagged with Myc. We transfected Nthy cells with the wild-type and P160L mutant then compared the distribution and location of the protein by double-label immunofluorescence (Fig 3F). The overexpressed wild-type $\beta 1$-tubulin coassembled incorporates into microtubules, in contrast to $\beta 1$-tubulin P160L (absence of incorporation). Thus, the P160L mutation clearly affects the capacity of $\beta 1$-tubulin to incorporate into microtubules, which is most likely related to defects at the structural level, thus forming a dysfunctional $\alpha / \beta$-tubulin dimer. 
In summary, all three TUBB1 mutations identified here likely lead to non-functional $\alpha / \beta$-tubulin dimers that cannot be incorporated into microtubules.

\section{Tubb1 knock-out in mice affects thyroid development and function}

The phenotype of patients bearing TUBB1 mutations suggests that $\beta 1$-tubulin may contribute to thyroid development and function. We

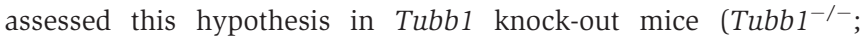
Schwer et al, 2001).

We found significant increases in expression levels of the other $\beta$-tubulin isoforms (Tubb2a, Tubb5, Tubb2b and Tubb3) in E17.5 thyroids of $\mathrm{Tubb1}^{-/-}$mice compared to wild-type mice (Appendix Fig S4A). Tubb2b and Tubb3 expression levels were also

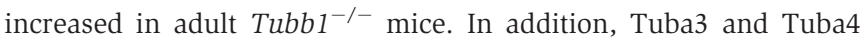

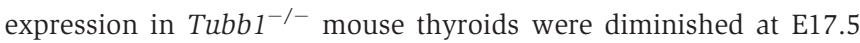
and in adults. Interestingly, these compensatory changes in expression levels seen in the thyroid gland mirror those described in platelets of $\mathrm{Tubb1}^{-/-}$mice (Appendix Fig S4A; Schwer et al, 2001).

\section{Thyroid gland development and differentiation}

Thyroid gland morphology in mice To study thyroid gland development from the early stages of budding and migration of the median anlage (MA) and ultimobranchial bodies (UB) to the late stages of differentiation, we used immunohistochemistry, surface quantification and quantitative PCR (Appendix Table S1). For most of the experiments, we used Nkx2-1 as a marker of progenitor and differentiated thyroid cells. At E9.5, thyroid anlage surface area and proliferation ratio were significantly greater in $\mathrm{Tubb1}^{-1-}$ mice than in wild-type mice (Fig 4A and B, and Appendix Table S2). At E11.5, thyroid migration was slightly delayed in three of six $T u b b 1^{-1-}$ embryos, a few Nkx2-1-positive cells were visible along the migration tract of the mutants, and thyroid surface area was not different between mutant and wild-type embryos (Fig 4A and B, and Appendix Table S2). During late thyroid development, at E13.5, fusion of the median anlage and ultimobranchial bodies was slightly delayed in three of four $\mathrm{Tubb}^{-/-}$embryos (Fig 4A and

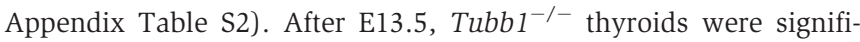
cantly hypoplastic (Fig 4A and B). Moreover, at E17.5, a supplementary pyramidal lobe was visible near the normal lobe in two of six embryos (Fig 4A). In keeping with this finding, patient P3 in family 1 described above, who was homozygous for the p.P160L mutation, had a hypoplastic thyroid with a pyramidal lobe. In sum, the thyroid phenotype of $T u b b 1^{-1-}$ mice indicates that $\beta 1$-tubulin is required for normal thyroid migration and morphology.

Thyroid gene ontogeny in mice We used quantitative PCR to assess the ontogeny of genes involved in thyroid function and development (Fig 4C). Expression levels of mRNAs for thyroglobulin (Tg), thyroid peroxidase (Tpo), TSH receptor (Tshr) and calcitonin (Calca), late differentiation markers, were significantly decreased at E15.5, as were those of Tg, Tpo and Calca at E17.5, in Tubb1 $1^{-1-}$ compared to wild-type embryos. Significant decreases were also

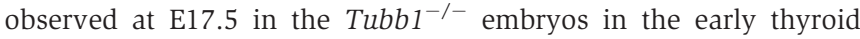
development markers Nkx2-1, Pax8 and Foxe1. These results indicate impaired thyroid gland development and differentiation in Tubb1 $1^{-1-}$ mice.

Endocrine signature at completion of thyroid gland development T4-positive thyroid surface area relative to total thyroid surface area was shown by immunohistochemistry to be signifi-

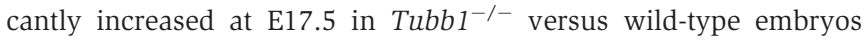
(Fig 4D). Calcitonin-positive thyroid surface area relative to total thyroid surface area tended to be greater in the mutants $(P=0.19)$. Thus, final thyroid differentiation was abnormal in Tubb1 $1^{-1-}$ embryos, with increases in intrathyroidal T4 that probably reflected impaired hormone secretion.

These results establish a role for $\beta 1$-tubulin in thyroid development and differentiation.

\section{Thyroid gland function and structure in adult mice}

We compared thyroid hormone status in adult $\mathrm{Tubb1}^{-/-}$and wild-type mice. At 3 months of age, serum TSH levels were

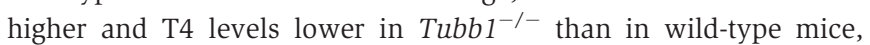
suggesting hypothyroidism in the mutants (Fig 5A). These results are consistent with the finding of $\mathrm{CH}$ in our patients carrying TUBB1 mutations.

Furthermore, we examined thyroid structure in adult mice ( 3 months of age). Surface area was not different between the

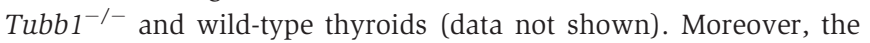
thyroid tissue was disorganized in the mutants with large regions without organized follicles (Fig 5B). When we used electron microscopy to examine thyrocyte ultrastructure (Fig 5C), we found larger

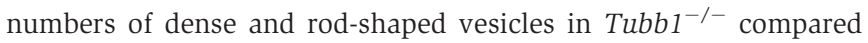
to wild-type thyrocytes. These data were consistent with impaired T4 release responsible for hypothyroidism. Marked endoplasmic reticulum (ER) dilation was seen in $\mathrm{Tubb}^{-1-}$ compared to wildtype thyrocytes (Fig 5C), indicating ER stress, which was confirmed by the findings of increased Chop and XBPs expression by quantitative PCR and of increased Chop protein levels by Western blotting in Tubb1 ${ }^{-/-}$thyroids (Fig 5D). By immunohistochemistry, the ER

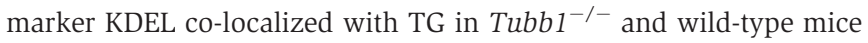
but was especially abundant in disorganized areas of adult Tubb1 $1^{-/-}$ thyroids (Fig 5E).

These data indicated partial thyroid tissue disorganization, vesicle accumulation and ER stress in $\mathrm{Tubb1}^{-1-}$ mice.

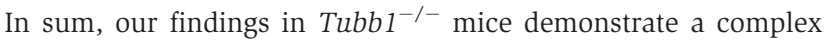
mechanism of hypothyroidism involving early abnormal proliferation of progenitors, delayed thyroid migration, defective thyroid differentiation, impaired thyroid hormone release and structural disorganization of the thyroid tissue. The abnormal thyroid migration may explain the thyroid phenotype found in patients carrying TUBB1 mutations, especially those with thyroid gland ectopia.

\section{Analysis of platelets from patients bearing TUBB1 mutations}

Until now, $\beta 1$-tubulin expression had been reported only in the megakaryocyte lineage (Patel et al, 2015). TUBB1 mutations have been previously reported to cause macrothrombocytopaenia (Kunishima et al, 2009, 2014; Fiore et al, 2017). We therefore studied the platelets of the above-described patients with TUBB1 mutations and thyroid gland abnormalities. 
A
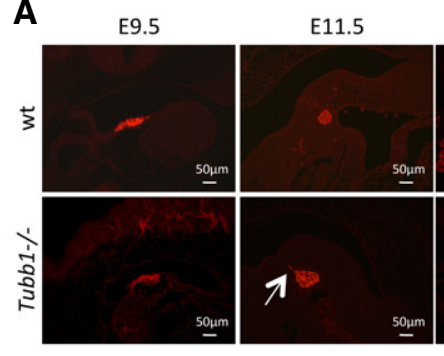

E13.5

B
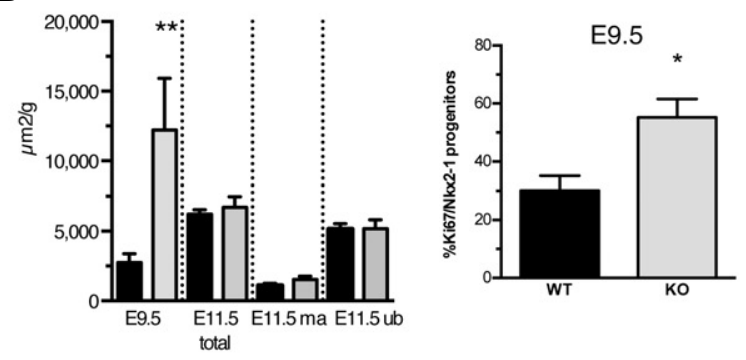

C

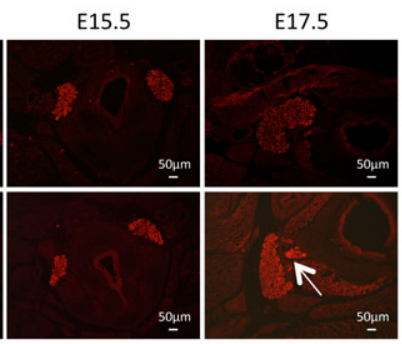

50um

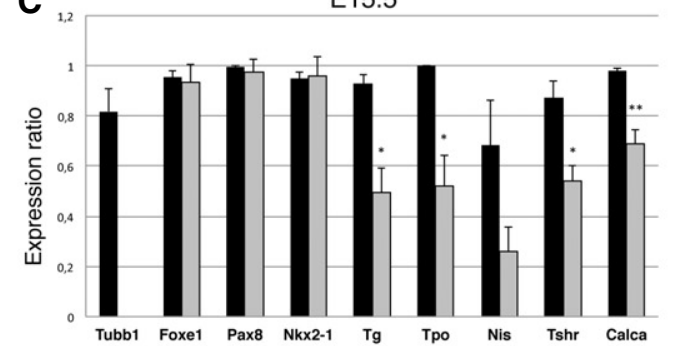

E17.5

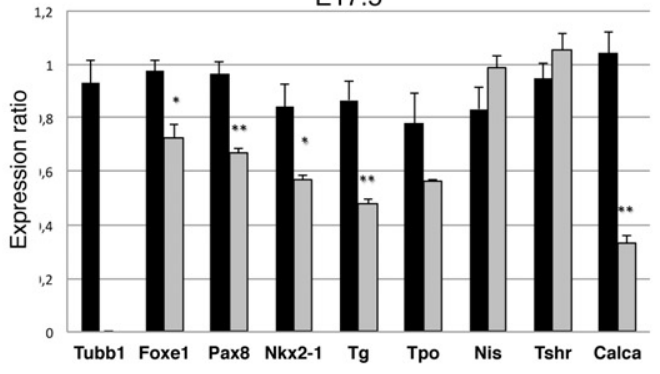

D
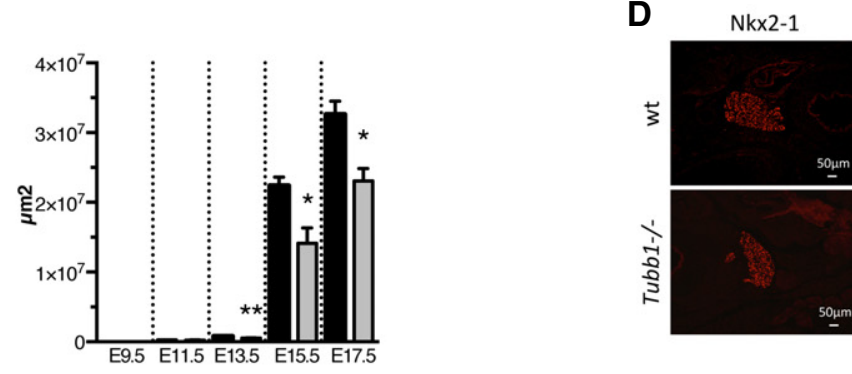

T4
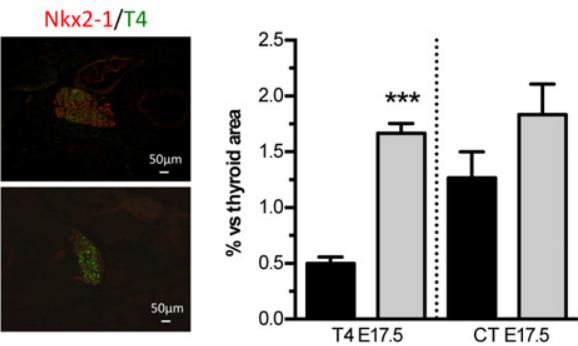

Figure 4. Tubb1 ${ }^{-1-}$ mice have abnormal thyroid development.

A Thyroid morphology was investigated using Nkx2-1 staining at E9.5 and E11.5 (sagittal sections) and at E13.5, E15.5 and E17.5 (transverse sections) in wild-type (wt) and Tubb1 ${ }^{-1-}$ littermates. Nkx2-1 was used as a marker of early thyrocyte progenitors and differentiated thyrocytes. Delays in thyroid migration and in fusion of the median anlage to the ultimobranchial bodies were observed at E11.5 and E13.5 (arrow), respectively, in Tubb1 ${ }^{-1-}$ mice. At E11.5, stained migrating thyroid cells were visible along the tract only in Tubb1 ${ }^{-1-}$ littermates (arrow). At E17.5, a pyramidal lobe was visible in Tubb1 ${ }^{-1-}$ littermates (arrow). tr: trachea; ub: ultimobranchial body. Scale bar: $50 \mu \mathrm{m}$.

B Total thyroid surface area $\left(\mu \mathrm{m}^{2}\right)$ was quantified using Nkx2-1 staining at each embryonic stage. Top: thyroid surface area normalized for weight of each embryo. Four mice were analysed per genotype at E9.5 and five mice at E11.5. Bottom: thyroid surface area in $\mu \mathrm{m}^{2}$. Four mice were analysed per genotype at E9.5, five at E11.5, six at E13.5, four at E15.5 and three at E17.5. Right: proliferation ratio calculated as the proportion of Nkx2-1-positive cells labelled with Ki67 at E9.5 in wt and Tubb1 ${ }^{-1-}$

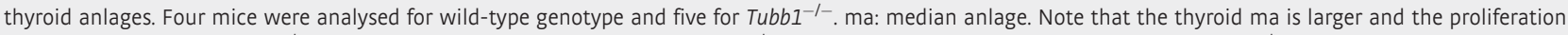

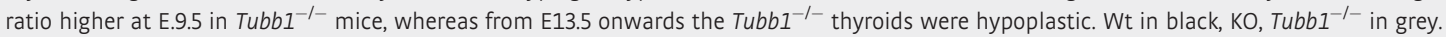

C Thyroid marker expression by quantitative PCR in thyroid tissue at E15.5 and E17.5 in Tubb1 ${ }^{-1-}$ versus wt mice normalized for peptidylprolyl isomerase A. Note the decreases in thyroid differentiation markers at E15.5 and E17.5 and also in thyroid transcription factors at E17.5. Four mice were analysed per genotype at E15.5 and three at E17.5.

D Staining of Nkx2-1 and T4 at E17.5 and surface area quantification demonstrating T4 retention in Tubb1 ${ }^{-1-}$ versus wt thyroids. The data are the percentage of T4 or calcitonin (CT) surface area versus total thyroid area (estimated from the Nkx2-1-stained surface area). Three mice were analysed per genotype. Scale bar: $50 \mu \mathrm{m}$.

Data information: Results are reported as mean \pm SEM. Student's $t$-test, ${ }^{*} P<0.05,{ }^{* \star} P<0.01$ and ${ }^{* * *} P<0.001$.

\section{Haematological parameters (Fig 1)}

Thrombocytopaenia was a feature in patients with TUBB1 mutations studied by other groups (Kunishima et al, 2009, 2014; Fiore et al, 2017). However, when we used an automated haematology analyser to measure the platelet count and mean platelet volume (MPV) in our seven patients, we consistently found normal platelet counts. In contrast, MPV was above the normal range in P1 and near the upper limit of normal in P4 and P7. A blood smear analysis (Fig 6A) demonstrated variations in platelet size with the presence of macroplatelets. Moreover, parents I.1 and I.2 of family F1 had MPV values at the top of the normal range $(10.2$ and $11.3 \mathrm{fl}$, respectively; normal, 7.5-11.2 fl) with many macroplatelets. Electron microscopy confirmed the large platelet size (Fig 6B).

The increased platelet size could be related to defects in their biogenesis. To study this, we cultured megakaryocytes from patients P1 and P4 from peripheral blood progenitors and analysed proplatelet formation (Fig 6C). Interestingly, shaft thickness and coiled element diameter of the future platelets were significantly increased compared to controls, indicating that the TUBB1 mutations affected proplatelet formation and platelet size. 
A
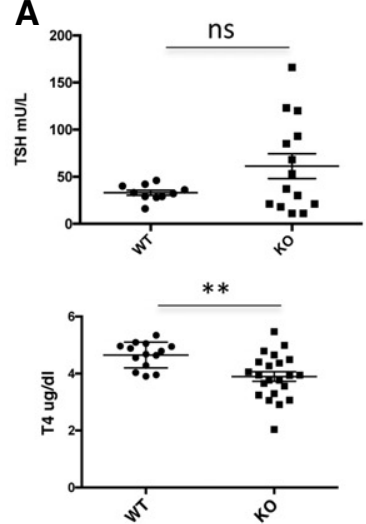
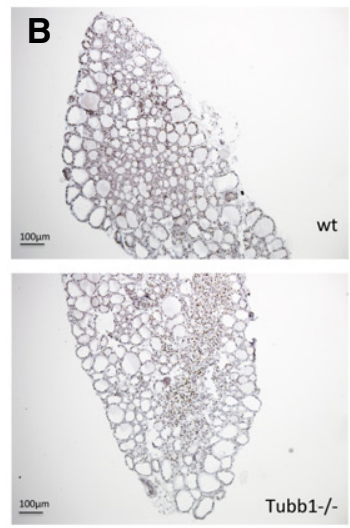

C
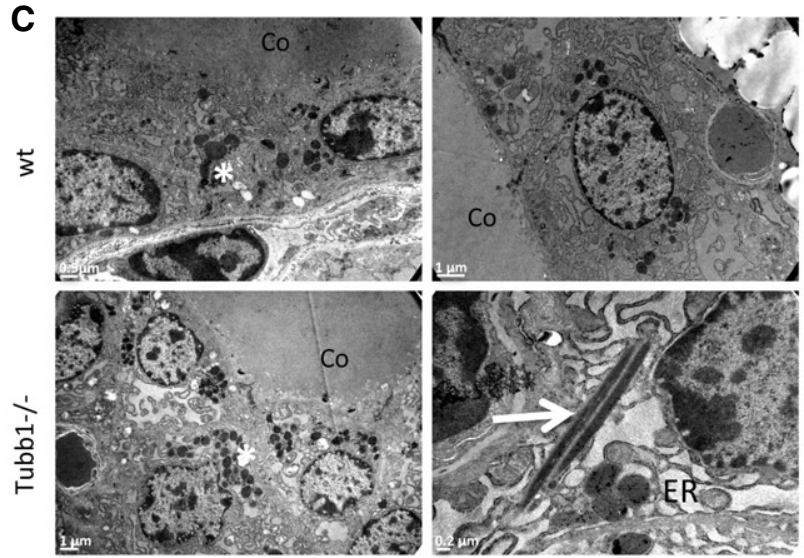

D

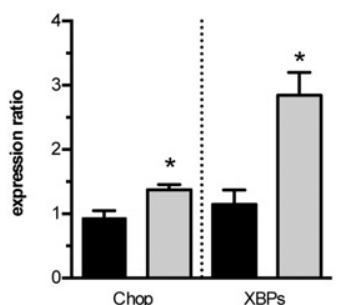

XBPs

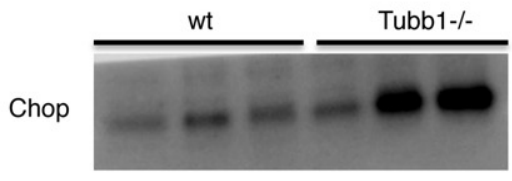

Actin
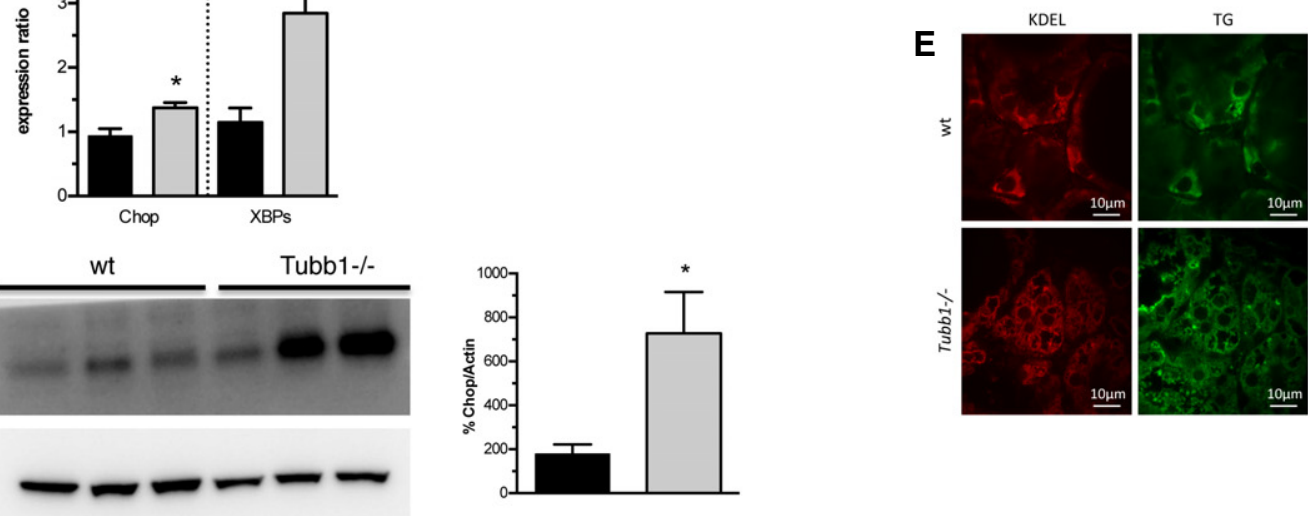

KDEL/TG

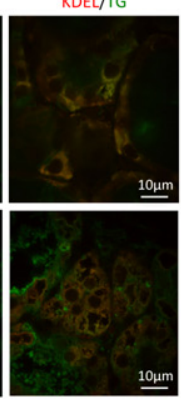

Figure 5. Tubb1 $^{-1-}$ mice have altered thyroid function with impaired hormone secretion.

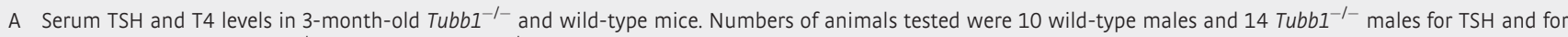
14 wild-type and $22 \mathrm{TubbI}^{-1-}$ males for T4. Tubb1 $1^{-1-}$ mice had hypothyroidism with elevated TSH and decreased T4 versus wild-type mice.

B Nkx2-1 (in brown) immunostaining of adult thyroid tissue. Note the disorganization of the thyroid tissue in Tubb $1^{-1-}$ versus wild-type mice.

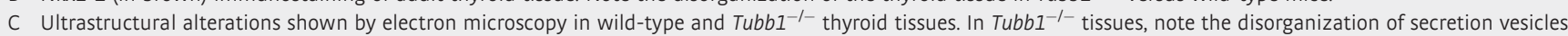
(white asterisks) and rods of identical density to secretion vesicles (white arrow). The ER is considerably dilated in Tubb1 ${ }^{-1-}$ thyrocytes. Representative views. Scale bars at the bottom left for each view. Co: colloid.

D ER stress in Tubb1 ${ }^{-1-}$ thyroid tissue. Top: Chop and XBPs expression by quantitative PCR in adults, normalized to peptidylprolyl isomerase A. Experiments with four tissues per stage for each genotype; wt in black and Tubb1 $1^{-1-}$ in grey. Bottom: Chop and Actin protein expression by Western blotting in three representative wild-

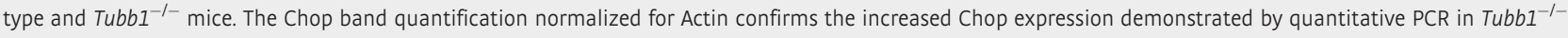
versus wild-type thyroids. All lanes are from the same blot, which was cut where indicated.

E Co-immunostaining of endoplasmic reticulum (ER) marker (KDEL, in red), thyroglobulin (Tg in green) and both merged (from left to right) in adult thyroid tissue. Note the thyrocyte disorganization in Tubb1 ${ }^{-1-}$ mice.

Data information: Results are reported as mean \pm SEM. Student's $t$-test, ${ }^{\star} P<0.05,{ }^{\star *} P<0.01$.

Source data are available online for this figure.

Functional analysis of human platelets

We first quantified $\beta 1$-tubulin expression in the platelets of our patients and found significant decreases $(P<0.001)$ of $45.0 \pm 3.7 \%$ and $36.5 \pm 3.4 \%$ in $\mathrm{P} 1$ and $\mathrm{P} 3$ (F1), respectively; $45.0 \pm 3.8 \%$ in $\mathrm{P} 4$ (F2); and $29.1 \pm 3.3 \%$ and $30.6 \pm 6.2 \%$ in P6 and P7 (F3), respectively. Expression of $\alpha$-tubulin was normal (Fig 7A). The $\beta 1$ tubulin antibody used in our study detects only the C-terminal part of the protein. Consequently, in the patients of families F2 and F3, whose mutations created premature stop codons, only the wild-type protein was detected. In the F1 patients, who were homozygous for the mutation, the results suggested either diminished protein expression or protein instability.

Then, to investigate whether the TUBB1 mutations affected platelet function, we assessed platelet activation by flow cytometry using a specific monoclonal antibody (PAC1), which recognized the active conformation of the integrin $\alpha_{\mathrm{II}} \beta_{3}$, a platelet activation marker. PAC1 binding was significantly increased in P1 and P3, indicating abnormal platelet activation, whereas in P6 and P7 (F3) $\alpha_{\mathrm{IIb}} \beta_{3}$ activations were comparable to those in controls (Fig 7B). We were unable to evaluate P4 (F2). 


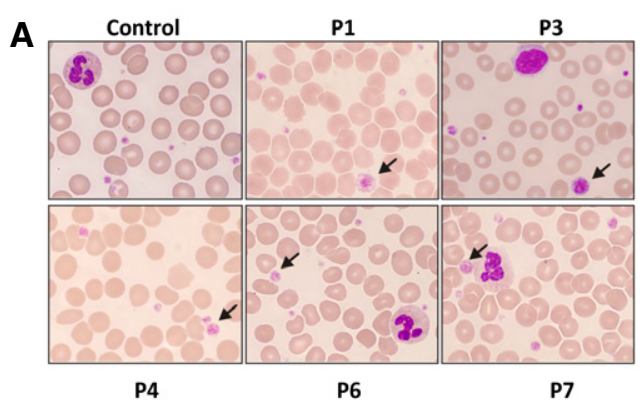

B
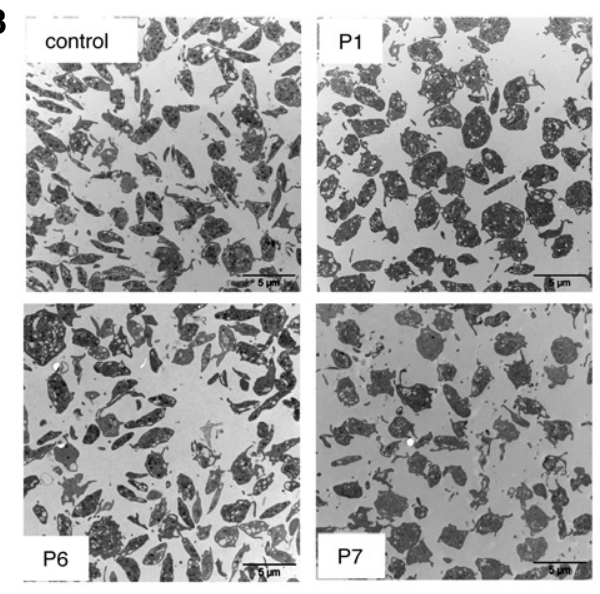

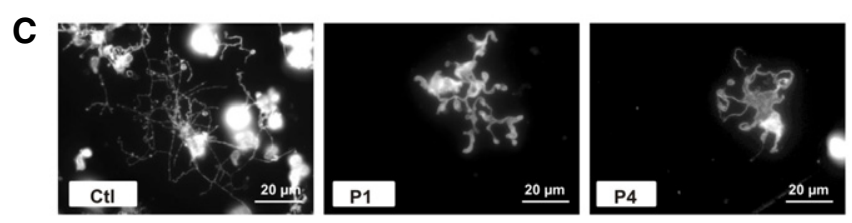

Shaft thickness
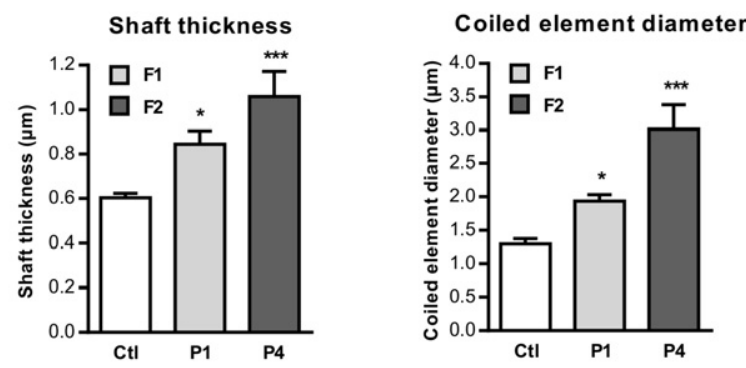

Figure 6. TUBB1 mutations induce abnormal platelet formation or macroplatelets.

A Representative blood smears of patients with TUBB1 mutations (P1 and P3 homozygous for p.P160L, P4 heterozygous for p.Y106X, and P6 and P7 heterozygous for c.35delG) after May-Grünwald-Giemsa staining. Variable platelet size in patients was observed with all three mutations with the presence of macroplatelets (arrows).

B Transmission electron microscopy (TEM) analysis of platelet ultrastructure in the patients. The results confirm the abnormal platelet morphology.

C Representative images of proplatelet formation in P1 and P4. Cultured control and patient megakaryocytes (MKs) after thrombopoietin-induced differentiation were spread over a BSA-coated coverslip on day 10 . On day 13 or 14 , MKs were fixed (4\% paraformaldehyde) and proplatelet structure was observed after $\beta$-tubulin staining. Scale bars: $20 \mu \mathrm{m}$. Only P1 and P4 could be studied. The graphs show the mean \pm SEM of the shaft thickness of proplatelet extensions (left) and the diameter of coiled elements (right) of the future proplatelets, both of which were significantly increased in the patients, indicating that the TUBB1 mutations (homozygous p.P160L and heterozygous p.Y106X) affected proplatelet morphology. Statistical significance was determined by one-way ANOVA, followed by Dunnett's multiple comparisons test ( ${ }^{\star} P<0.05,{ }^{\star * *} P<0.001 ; 20-60 \mathrm{MKs}$ were analysed/patient).

Finally, we investigated the aggregations of washed platelets upon activation by ADP or collagen, two key platelet agonists (Fig 7C). Aggregation in response to ADP was normal in P6 and P7 (F3) but increased in P1 and P3 (F1) and in P4 (F2) compared to controls. Similarly, collagen-induced platelet aggregation was normal in $\mathrm{P} 6$ and $\mathrm{P} 7$ but was increased in P1 and P4, even with low doses. These abnormal platelet aggregations were not related to hypothyroidism or patient's treatment by L-thyroxine, since they were not found in three patients with thyroid ectopy not harbouring TUBB1 mutations and under the same treatment (Appendix Fig S5). Moreover, it should be noted that these results were confirmed by at least three independent investigations for P1 and P3 and two for P6. The other patients were studied once. None of the nine controls exhibited similar hyperaggregation profile.

In summary, these results indicate that p.P160L (F1), p.Y106X (F2) and c.35delG (F3) TUBB1 mutations affect $\beta 1$-tubulin expression in platelets and result in abnormally large platelet size, probably as a consequence of proplatelet abnormal formation. Moreover, the p.P160L mutation induces significant platelet activation in resting condition and hyperaggregation in response to agonists, whereas the c.35delG mutation does not seem to affect platelet function.

\section{Discussion}

We identified three TUBB1 mutations in patients with TD and macroplatelets. In a consanguineous family (F1), two females with $\mathrm{CH}$ and thyroid gland ectopia had the same homozygous TUBB1 mutation, and their brother had thyroid gland hypoplasia with normal function. Of 270 patients with $\mathrm{CH}$ and $\mathrm{TD}$, two probands from unrelated families had heterozygous TUBB1 mutations. All patients with heterozygous or homozygous TUBB1 mutations had TD and macroplatelets. Thyroid gland ectopia was the most common form of TD, but mild thyroid asymmetry, thyroid hypoplasia and hemithyroid were also seen.

This is the first time that $\mathrm{CH}$ with TD was associated with macroplatelets, and we demonstrate in our study that TUBB1 mutations are the common cause. $\beta 1$-tubulin expression has heretofore been 

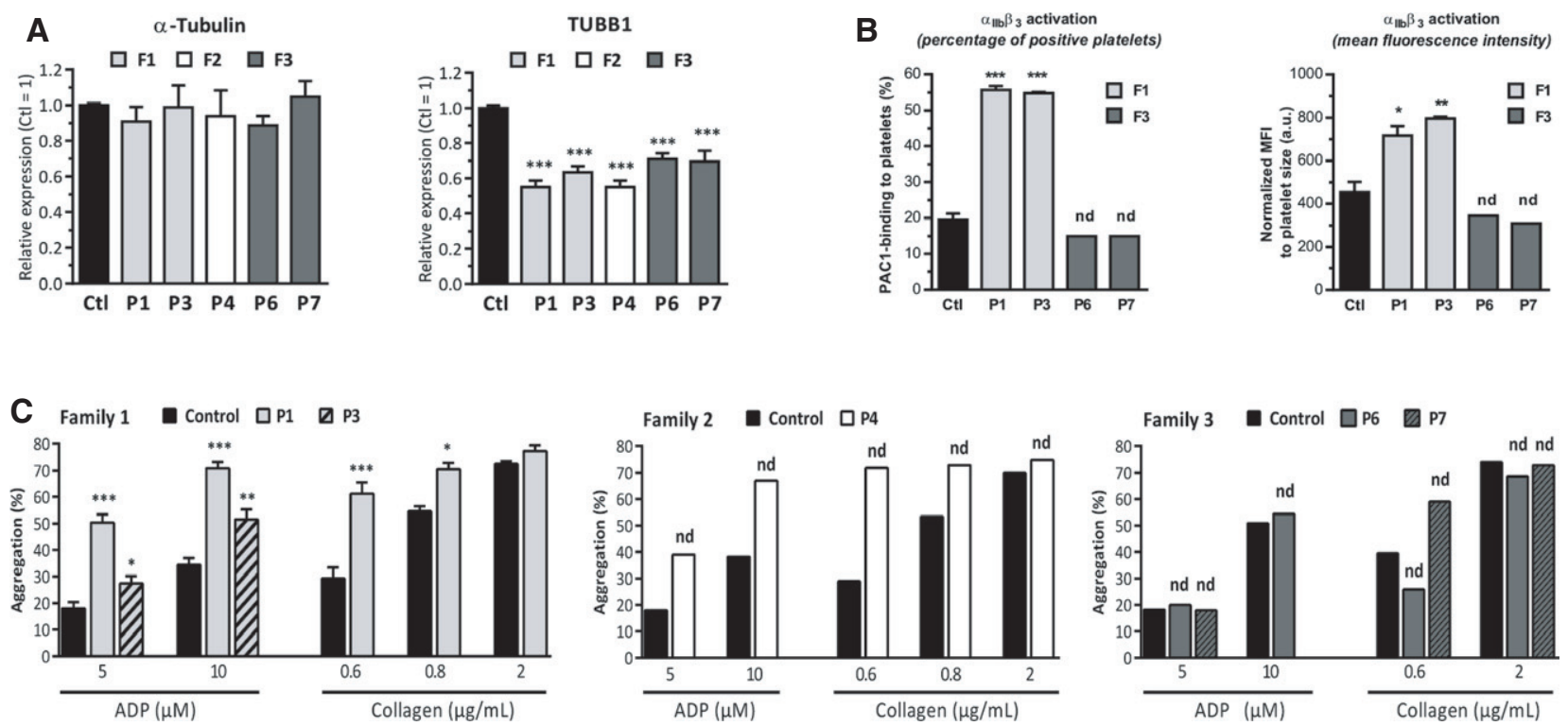

Figure 7. TUBB1 mutations alter protein expression in platelets and platelet functions.

A Relative expression of $\alpha$-tubulin and $\beta 1$-tubulin in platelets from P1 and P3 (homozygous p.P160L), P4 (heterozygous p.Y106X), and P6 and P7 (heterozygous C.35delC) was quantified by Western blotting using specific antibodies against $\alpha$-tubulin or $\beta 1$-tubulin. With both mutations, $\alpha$-tubulin expression was normal but $\beta 1$-tubulin expression was significantly decreased. The $\beta 1$-tubulin antibody recognizes $\mathrm{C}$-terminal domain.

B $\quad \alpha_{11} \beta_{3}$ integrin activation was evaluated by flow cytometry (\% of positive platelets and mean fluorescence intensity) in whole blood from P1 and P3 (homozygous p.P160L) and from P6 and P7 (heterozygous c.35delC); P4 could not be studied. While no $\alpha_{\| 1} \beta_{3}$ integrin activations were detected in P6 and P7 (family 3), P1 and P3 (family 1 ) exhibited a significant increase in $\alpha_{\| b} \beta_{3}$ activation, indicating basal platelet activation.

C Aggregation of washed platelets induced by ADP $(5$ or $10 \mu \mathrm{M})$ or collagen $(0.6,0.8$ or $2 \mu \mathrm{g} / \mathrm{ml})$ was evaluated in at least one member of each family. Patients from families 1 and 2 had increased platelet aggregation in response to low doses of agonist versus controls, suggesting that TUBB1 affected by homozygous p.P160L and heterozygous p.Y106X mutations affected platelet function. Platelet aggregation to both agonists was normal in both patients from family 3 (heterozygous c.35delG).

Data information: Results are reported as mean \pm SEM. Family F1, $n=3$ independent experiments; Family F2, $n=1$ experiment; Family F3, $n=2$ independent experiments. Statistical significance was determined by one-way ANOVA, followed by Dunnett's multiple comparisons test ${ }^{*} P<0.05$, ${ }^{* *} P<0.01$, ${ }^{* * *} P<0.001$, nd: statistical significance not determined because $n \leq 2$ ).

described as confined to megakaryocytes and platelets. Our findings demonstrate that $\beta 1$-tubulin is also expressed in developing and adult thyroid tissue. Furthermore, $\mathrm{CH}$ and $\mathrm{TD}$ were found in humans

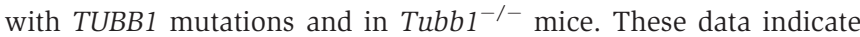
that normal thyroid development and function require $\beta 1$-tubulin incorporation into the microtubule network. Furthermore, our patients with TUBB1 mutations had normal platelet counts but abnormal platelet morphology, and two of the three mutations (p.P160L and p.Y106X) were associated with abnormal platelet function. In contrast, in previous studies, humans with TUBB1 mutations

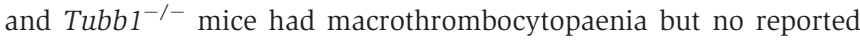
thyroid disorders (Kunishima et al, 2009, 2014; Fiore et al, 2017).

The previously reported TUBB1 mutations affect the intermediate or $\mathrm{C}$-terminal domain of $\beta 1$-tubulin, whereas the three novel mutations described here modify the N-terminal domain. However, during the drafting of this work, Bastida et al (2018) published a genetic analysis of a cohort of patients with inherited platelet disorders where they identified four novel TUBB1 mutations: two in the $\mathrm{C}$-terminal domain and two in the N-terminal domain, including the same mutation that we described here for family F3, c.35delG. In their study, the patient with the c.35delG mutation had a thrombocytopaenia, which differs from the normal platelet count of our family F3. This discrepancy could be explained by another factor, different from the TUBB1 mutation, which could induce thrombocytopaenia.
Two of our described mutations gave stop codon earlier but not the p.P160L mutation. The p.P160L protein expression was decreased in the platelets of patients, but the hypothesis of the protein instability was not conclusive in vitro experiments (data not shown). Others mechanisms should be considered. Strikingly, all three mutations are loss-of-function mutations: two of the mutations lead to truncated $\beta 1$-tubulin, which cannot form functional $\alpha / \beta$-tubulin dimers and thus cannot be incorporated into microtubules. The third is a missense mutation (P160L), which, despite expressing full-length $\beta$ tubulin, still does not allow the formation of functional $\alpha / \beta$-tubulin dimers to be integrated into the microtubules network. Hence, the three TUBB1 mutations were deleterious for the $\beta 1$-tubulin function, which is confirmed on the functional level, as their phenotypes are

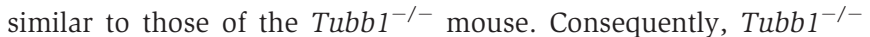
mice emerged as a useful tool for studying the impact of impaired $\beta 1$ tubulin function on the thyroid gland. Considering the hypothyroidism phenotype present only in $N k \times 2-1^{-1-}$ or $\mathrm{Pax}^{-/-}$or double Nkx2-1 $1^{+/-} \mathrm{Pax} 8^{+/-}$mice, we decided to study directly the knock-out mice (Kimura et al, 1996; Mansouri et al, 1998; Amendola et al, 2005). Haploinsufficiency of one of these critical genes for thyroid development causes hypothyroidism in humans, whereas in mice, the homozygous deletion leads to thyroid phenotype.

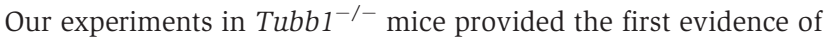
a role for $\beta 1$-tubulin in TD and hypothyroidism. The thyroid glands 


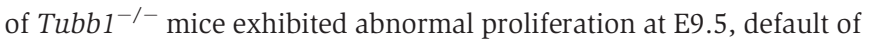
migration at E11.5 and E13.5, and hormone secretion failure at E17.5 and adulthood. All these mechanisms require normal microtubule organization and function. The idea that microtubule functions are fine-tuned and thus adapted to specific cellular role by the expression of specific tubulin isotypes is an emerging concept known as the "tubulin code" (Janke, 2014). Tubulin mutations have been more and more linked to different human disorders, in particular to neurological disorders (Chakraborti et al, 2016). Microtubules are essential for mitosis to unfold normally (Prosser \& Pelletier, 2017). Here, we show that invalidation of $\beta$-tubulin isotype Tubb1 perturbs mitosis of progenitors at E9.5, thus inducing hyperproliferation. Mitotic phenotypes have already described during neuronal development in mice with loss of another $\beta$-tubulin isotype, Tubb5 (Breuss et al, 2012). Moreover, normal thyroid development requires that progenitor proliferation occurs during a specific time window (Nilsson \& Fagman, 2017). Early proliferation at E9.5, as described by our group in $H e s 1^{-/-}$mice (Carre et al, 2011), results in abnormal thyroid development, probably via impairment of the pool of progenitors dedicated to thyroid development. Furthermore, microtubules are required for cell orientation during migration (Ladoux et al, 2016).

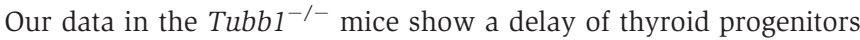
cell migration during thyroid development, which again is mirrored by $\mathrm{Tubb5}^{-1-}$ that show impaired neuronal migration (Breuss et al, 2012). In our patients with TUBB1 mutations, thyroid gland ectopia was the most common form of TD (4/7 patients), further supporting a role for $\beta 1$-tubulin in thyroid gland migration. The delay of thyroid bud migration could be reminiscent of the dual ectopy seen in $9 \%$ of $\mathrm{CH}$ due to ectopy (Wildi-Runge et al, 2012). Finally, endosome/lysosome trafficking is taking place on microtubules (Huotari \& Helenius, 2011; Raiborg et al, 2015; Bonifacino \& Neefjes, 2017). Endosome-tolysosome transport of thyroid hormones via a vesicular transport system has been described in the thyroid gland (Rousset et al, 2015; Carvalho \& Dupuy, 2017). A proper thyroid microtubule integrity is required for thyroid hormone secretion established by previous studies done in the 1970s (Nève et al, 1972; Wolff \& Bhattacharyya, 1975). While so far nothing is known on the precise role of tubulin isotypes and microtubules organization in trafficking of thyroid hormone vesicles, there is strong evidence that transport along microtubules is affected by the tubulin code (Nirschl et al, 2016). Our data are the first to demonstrate that a specific tubulin isotype, $\beta 1$-tubulin, is required for proper vesicle trafficking and thyroid hormone release into the bloodstream, and they extend our knowledge about the association between microtubules and secretion vesicles in the thyroid gland. Furthermore, we have shown an increased

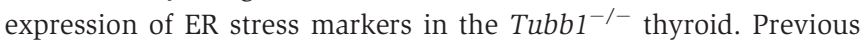
studies demonstrated ER stress with activation of the unfolded protein response in association with reduced thyroglobulin (TG) synthesis and TG accumulation within the ER (Gaide Chevronnay et al, 2015; Di Jeso \& Arvan, 2016). We can conclude that thyroid dysgenesis is a feature in TUBB1 mutation carriers and in Tubb1 $1^{-1-}$ mice. The data on variable degrees of hypothyroidism in humans corroborate with the thyroid function data in mice as the variable TSH values have been found.

$\beta 1$-tubulin is the most divergent isotype of tubulin, and its incorporation into microtubules is expected to change the properties of these filaments. Losing $\beta 1$-tubulin thus certainly alters microtubule properties, and our data provide strong evidence that these particular properties are necessary for adequate thyroid development and function.

Our patients with TUBB1 mutations had macroplatelets. This abnormal platelet size is further evidence that TUBB1 mutations adversely affect platelet morphology probably as a consequence of proplatelets formation. In these previously described mutations, only the p.R318W mutation has been investigated in platelet aggregations and it did not induce defect of platelet function (Kunishima et al, 2009). For the other mutations (Kunishima et al, 2014; Johnson et al, 2016; Fiore et al, 2017; Bastida et al, 2018), no bleeding tendency have been reported. Here, our patients with p.P160L and p.Y106X mutations exhibited an unexpected increase in platelet functions, raising the question whether these platelet function abnormalities result in clinical symptoms such as thrombosis requires evaluation, particularly in older patients who may be at increased risk. Thyroid dysfunction can affect the haemostatic balance (Squizzato et al, 2007; Kyriakakis et al, 2016), with clinical effects that vary across thyroid disorders (Franchini et al, 2010). Moreover, MPV correlated positively with the TSH level (Kim et al, 2013). A TUBB1 mutation screening study in patients with hypothyroidism and altered MPV and/or a history of thrombotic disease would be of great interest. Therefore, patients bearing a TUBB1 mutation with cardiovascular risk factors could be monitored and the question arises whether antiplatelet drugs might be effective as prophylaxis (Kuhli-Hattenbach et al, 2017). Conversely, thyroid function and morphology assessment could be suggested in patients with congenital macrothrombocytopaenia.

The c.35delG mutation in P6 and P7 did not cause hyperaggregation. The patients with the c.35delG mutation had the same thyroid phenotype as other families with other mutations. At least one patient per family had thyroid gland ectopia with $\mathrm{CH}$. Neither thyroid nor platelet phenotype severity correlated with the type of mutation. Further investigations are needed to elucidate the difference between the effects of c.35delG and p.P160L/p.Y106X on platelet function.

The common feature in TUBB1 mutation carriers is thyroid dysgenesis and abnormal platelet morphology. TUBB1 mutations constitute a model of dominant inheritance of $\mathrm{CH}$ with $\mathrm{TD}$. Most known mutations responsible for TD and previously described TUBB1 mutations causing macrothrombocytopaenia are also dominant (Kunishima et al, 2009, 2014; Guillot et al, 2010; Ramos et al, 2014; Carré et al, 2017; Fiore et al, 2017). Our data indicate high penetrance for platelet alterations and incomplete penetrance with variable expressivity for TD, ranging from TD without $\mathrm{CH}$ to $\mathrm{TD}$ with $\mathrm{CH}$ and leading to variable types of TD (ectopia, hypoplasia, hemithyroid or asymmetric thyroid gland). No patient had athyreosis. Indeed, in the described familial pedigrees, some carriers have mild or no thyroid phenotype, suggesting that the TUBB1 germline mutation may be necessary to be affected by CHTD but it is probably not sufficient to display the phenotype. A second hit (such as a somatic mutation in the thyroid or an epigenetic defect) could be the additional prerequisite to express the disease. Furthermore, the hypothesis of random autosomal monoallelic expression in the thyroid could explain the difference in intrafamilial phenotypic variability in F3. These hypotheses are already documented in the literature for TD (Deladoëy et al, 2007; Magne et al, 2016). Finally, the genetics of TD remains complex with mutations in more than nine known genes and both classical and complex modes of inheritance, such as a suggested 
oligogenic model by Persani et al (de Filippis et al, 2017). The same genetic pattern of inheritance is also observed in other endocrinerelated disorders such as congenital hypogonadotropic hypogonadism (Boehm et al, 2015) or in the more complicated genetic model of Bardet-Biedl syndrome (Muller et al, 2010).

TUBB1 mutations were found in only $1.1 \%$ of our cohort with $\mathrm{CH}$ and $\mathrm{TD}$ patients. The Burden test showed enrichment in rare TUBB1 variants carriers in the cohort versus controls. Our data increment the number of predisposing genes for thyroid dysgenesis but with a novel phenotype associating platelet disorder.

Taken together, our data indicate heretofore unsuspected roles for a specific isotype of $\beta$-tubulin, Tubb1, in thyroid development and function, while also confirming its importance for microtubule integrity and platelet function. Loss-of-function of TUBB1 mutations impairs $\beta 1$-tubulin incorporation into microtubules. Our results confirm that normal thyroid-cell proliferation and thyroid migration are essential to thyroid gland development. Thyroid hormone secretion requires $\beta 1$-tubulin incorporation into the microtubules, suggesting a specific function of this tubulin isotype in intracellular transport of vesicles. Thus, our work provides novel insights into the role of the Tubb1 isotype in thyroid physiopathology and in platelet function and therefore expands the spectrum of the rare paediatric diseases related to tubulin mutations and microtubule dysfunction.

\section{Materials and Methods}

\section{Patients}

We have a large study cohort of patients with congenital hypothyroidism due to TD. Patients have been diagnosed with primary $\mathrm{CH}$ during the first days after birth by the systematic neonatal screening in France $(\mathrm{TSH}>15 \mathrm{mU} / \mathrm{l}$ ). Diagnosis of $\mathrm{CH}$ was confirmed with a control blood sample during the first weeks of life and imaging tests (thyroid ultrasonography and thyroid scintigraphy). A consanguineous family in which two children had TD and $\mathrm{CH}$ and another had small thyroid gland with normal thyroid function was initially studied. Subsequently, genetic testing was performed in 270 patients (184 girls and 86 boys) with $\mathrm{CH}$ and TD (ectopic thyroid gland, $n=167$; athyreosis, $n=77$; hemithyroid $n=20$; and thyroid hypoplasia, $n=6$ ). This study was approved by the review board (Ethics Committee, Ile de France, Paris, France: P11012-IDRCB 2012-A00797-36). The written informed consent forms were collected, and the experiments conformed to the principles set out in the WMA Declaration of Helsinki and the Department of Health and Human Services Belmont Report.

\section{Detection of mutations in humans}

Genomic DNA was isolated from whole blood. Exome capture and sequencing were performed at the genomics platform of the IMAGINE Institute. WES libraries were prepared from $3 \mu$ g genomic DNA per individual, which was sheared by ultrasonication (Covaris S220 Ultrasonicator, Woburn, MA, USA). Exome capture was performed using the SureSelect Human All Exon V6 Kit (Agilent Technologies, Santa Clara, CA, USA). The resulting libraries were sequenced on a HiSeq 2500 HT device (Illumina, San Diego, CA, USA) according to the manufacturer's recommendations. Paired-end $(2 \times 130)$ 76-bp reads were generated and mapped on the human reference genome. More than $97 \%$ of the exome was covered at least 30 times. Raw data were analysed as described (Gordon et al, 2013), using an in-house software system (Polyquery). The variant prioritization strategy was as follows: (i) selection of functional (protein-altering) variants (removal of intergenic and $3^{\prime} / 5^{\prime}$ UTR variants, non-splice-related intronic variants and synonymous variants); (ii) variants with a frequency below $1 \%$ in public databases (dbSNP, 1000 Genomes, EVS, ExAC; release date, January 2018); and (iii) variants previously identified in fewer than five individuals contributing 11,811 in-house exomes (Appendix Fig S1).

The HypothySeq NGS Panel included 78 genes known to be associated with $\mathrm{CH}$ (TD; dyshormonogenesis; defects in thyroid hormone $(\mathrm{TH})$ transport proteins; and inborn errors in TH membrane transport, metabolism or action) and candidate genes validated in animal models (mouse and zebrafish knock-out models) or by microarray assays but not yet validated in humans. This panel was previously validated using controls including samples from positive controls with known thyroid disease-causing mutations, to assess sensitivity (false-negative rate); and from healthy individuals screened by WES for another research study, to test specificity (false-positive rate). Genomic DNA libraries were created using SureSelectXT Target Enrichment Reagent Kit (Agilent Technologies) and subjected to custom-targeted DNA panel enrichment. In the 78 genes associated with $\mathrm{CH}, 1,006$ regions of interest were captured by the corresponding 120-bp cRNA baits, using SureDesign software (Agilent Technologies; Homo sapiens, hg19, GRCh37, February 2009). The 233,103-bp targeted DNA regions (protein-coding exons of the main isoform and supplementary coding exons of each gene, including 25bp flanking intronic sequences) were sequenced on Illumina HiSeq 2500 (Illumina). This step generated $2 \times 130$ paired-end reads. Bioinformatic analyses included alignment against the reference genome, variant calling and annotation, and copy number variation $(\mathrm{CNV})$ detection. All data were integrated in the dedicated interface Polydiag developed by the bioinformatics platform at the IMAGINE Institute to check coverage of the targeted regions, to sort and filter the called variants by impact and frequency, and to identify relevant candidate mutations and/or CNVs for molecular diagnosis.

Sanger sequencing was performed to validate and segregate the identified TUBB1 (NM_030773) variants (3500xL Genetic Analyzer, Thermo Fisher Scientific, Waltham, MA, USA) with listed primers in Table EV2.

\section{Burden test}

Rare variant burden testing was performed for the TUBB1 gene using the CAST collapsing method (Morgenthaler \& Thilly, 2007) in 270 patients with TD, including 193 with ectopia, hemithyroid or hypoplasia and 77 with athyreosis. Contrary to a single-variant association test, variants of the same gene were aggregated and considered as a whole. For each individual, the presence or absence of variants in the gene was noted. The aim of the method was then to count and compare the number of individuals carrying at least one potentially deleterious variant in the TUBB1 gene, in the two groups of individuals. To do so, a likelihood ratio test was performed to compare the TD patients with 406 Caucasian controls from the 1,000 Genomes project phase 3 (The 1000 Genomes Project Consortium, 2015). Disruptive in-frame, frameshift, missense, splice-acceptor, splicedonor, start-lost, stop-gained or stop-lost variants were considered 
deleterious. All deleterious variants with a minor allele frequency $<1 \%$ in the ExAC database r0.2 were included in the analysis. We first studied all 270 TD patients then only the 193 TD patients without athyreosis group.

\section{Human thyroid tissue samples}

After approval by our institutional review board of the experimental design and protocols, embryonic thyroid tissue was obtained from products of elective termination of pregnancy and adult thyroid tissues from patients undergoing thyroid surgery.

\section{Animals}

Tubb1 $1^{-1-}$ mice were previously generated by replacing exons 3 and 4, encoding amino acids 56-451, with a neomycin-resistance gene cassette as previously described (Schwer et al, 2001). Tubb1 $1^{+/-}$ mice on a mixed 129/Sv-BALB/c background were interbred with $\mathrm{C} 57 \mathrm{BL} / 6 \mathrm{~J}$ mice over ten generations to generate homozygous null mutants (Tubb1 ${ }^{-/-}$) with the C57BL/6J background (B6.CG- $\beta 1$ tubu$\left.\operatorname{lin}^{\mathrm{TM}}\right)$. All experiments were conducted in accordance with French regulations and were approved by the Strasbourg Regional Ethics Committee for animal experimentation (C.R.E.M.E.A.S., CEEA 35). Animals were housed in a temperature-controlled room on a 12 -h light/12-h dark cycle and had free access to food and water. All adult mice were male. Thyroids at different embryonic stages from E13.5 to E17.5 and adult thyroids at 3 months of age were obtained from

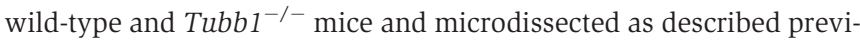
ously (Carre et al, 2011).

\section{Assays on mouse serum samples}

Aortic blood samples were collected from 3-month-old wild-type and $\mathrm{Tubb1}^{-1-}$ mice. Radioimmunoassays were used to measure serum TSH and serum total T4 after iodothyronine extraction (Dr. S. Refetoff, Chicago, IL, USA) as previously described (Pohlenz et al, 1999).

\section{Flow cytometry of mouse thyroid cells}

Mouse thyroid tissue from E17.5 embryos (15 pooled thyroids per sample) and adults (four thyroids per sample) were microdissected, cleansed of fat and connective tissue, and placed in ice-cold phosphate-buffered saline (PBS) containing $2 \%$ foetal calf serum (FCS). Cells were prepared and sorted by flow cytometry as previously described (Gawade et al, 2016). Briefly, single-cell suspensions were obtained by enzymatic digestion with $1 \mathrm{mg} / \mathrm{ml}$ collagenase/dispase and $2 \mu \mathrm{g} / \mathrm{ml}$ DNase I (Roche Diagnostics, Basel, Switzerland) at $37^{\circ} \mathrm{C}$ for $20 \mathrm{~min}$. The cells were then centrifuged with PBS containing $2 \%$ FCS and stained with cell surface markers for $20 \mathrm{~min}$. Finally, the cells were acquired on a BD FACSAria II flow cytometer (Becton Dickinson, Franklin Lakes, NJ, USA). The following monoclonal antibodies were used: EpCAM/CD326 (1:1,000, clone G8.8, \# 118216), PDGFRa/CD140a (1:400, APA5, \# 135906), CD45 (1:200, clone 30-F11, \# 103128) and Pecam/CD31 (1:400, clone 390, \# 102406) from BioLegend (San Diego, CA, USA); and CD41 (1:50, clone MWReg30, \# 553848, Becton Dickinson). The secondary antibody was goat anti-rabbit A647 from Life Technologies (1:2,000;
Carlsbad, CA, USA). Each pool of sorted cells was collected in RLT buffer from the Qiagen RNeasy MicroKit (Qiagen, Valencia, CA, USA) for RNA extraction experiments.

\section{RNA extraction and quantitative RT-PCR}

The thyroids were microdissected and immediately snap-frozen and stored at $-80^{\circ} \mathrm{C}$. Total RNA of sorted cells or thyroid tissue was isolated using the Qiagen RNeasy MicroKit or MiniKit (Qiagen). The Maxima First Strand cDNA Synthesis Kit (Thermo Fisher Scientific) was used for reverse transcription of $250 \mathrm{ng}$ of each RNA sample. The synthesized cDNA was diluted to $1 / 20$, and $5 \mu \mathrm{l}$ was used for each PCR. Each reaction consisted of TaqMan Universal PCR Master Mix or SybrGreen PCR Master Mix (Thermo Fisher Scientific) and primers. Peptidylprolyl isomerase A served as an endogenous control. Real-time PCR was performed using the QuantStudio 3 Real-Time PCR System (Thermo Fisher Scientific). The data were analysed using the comparative cycle threshold method and reported as the fold change in gene expression, normalized for a calibrator of value 1. Primers sequences for human TUBB1 were as follows: Forward GGGACGATGGACAGCATTCGAT and Reverse ACCTCTAGGACATTCTCGATCAGC. Primers sequences for mice $\alpha$ and $\beta$-tubulin are listed in Appendix Fig S4B.

\section{Immunohistochemistry and quantification}

Human or mouse tissues were fixed by immersion in 3.7\% buffered formalin then embedded in paraffin. Subsequently, $4-\mu \mathrm{m}$-thick sections were mounted on StarFrost adhesive slides (Knittel Glaser, Braunschweig, Germany) and processed for immunohistochemistry, as previously described (Carre et al, 2011). The primary antibodies were used at the following dilutions: rabbit antibody to human or mouse $\beta 1$-tubulin, 1:1,000 (donated by François Lanza), rabbit antiEcadherin, 1:100 (\# 610682, Becton Dickinson), mouse anti-TG, 1:100 (\# M0781, DakoCytomation, Glostrup, Denmark), rabbit antiNkx2-1, 1:2,500 (\#PA0100, Biopat, Italy), mouse anti-T4, 1:10,000 (clone BGN/0980/322, \# 8959-9831, AbD Serotec, Raleigh, NC, USA), rabbit anti-calcitonin, 1:400 (\# A0576, DakoCytomation), mouse anti-Ki67, 1:20 (\# 550609, Becton Dickinson) and rabbit antiKDEL, 1:1,500 (\# PA1-013, Thermo Fisher Scientific). The fluorescent secondary antibodies were Alexa Fluor 594 goat anti-rabbit and Alexa Fluor 488 goat anti-mouse antibodies (1:400, Thermo Fisher Scientific). The nuclei were stained using the Hoechst 33,342 fluorescent stain $(0.3 \mathrm{mg} / \mathrm{ml}$; Thermo Fisher Scientific). Photographs were taken using a fluorescence microscope (Leitz DMRB; Leica, Wetzlar, Germany) and digitized using a chilled 3CCD camera (C5810; Hamamatsu Photonics, Hamamatsu City, Japan).

The sections were then analysed using ImageJ 1.32s (freeware, www.rsbweb.nih.gov/ij) as previously described (Carre et al, 2011; Kariyawasam et al, 2015). The Nkx2-1-positive surface areas per section allowed us to draw the total thyroid surface area in $\mu \mathrm{m}^{2}$. The surface areas positive for calcitonin and T4, two markers of late thyroid differentiation, were normalized for total thyroid surface area. For stained surface quantification, we used one of every two sections at E9.5 and E11.5, one of every five sections at E13.5 and five sections per adult thyroid ( 3 months of age). We determined the surface area to obtain an estimate of the total stained surface for each thyroid and each marker. Proliferation of Nkx2-1-positive cells 
at E9.5 was estimated by counting Ki67-positive nuclei among Nkx2-1-positive cells on every other section throughout the entire tissue sample at E9.5. At least three thyroids were analysed per genotype. The results are reported as mean \pm SEM.

For Nkx2-1 staining of adult mouse thyroid glands, the first immunohistochemistry steps were as described above. After application of the primary antibody, the sections were incubated with biotinylated secondary antibody for $1 \mathrm{~h}$. Immunostaining was then performed using the Vectastain $\mathrm{ABC}$ Kit (Vector Laboratories, Burlingame, CA, USA) according to the manufacturer's instructions. The sections were then incubated in 3,3'-diaminobenzidine tetrahydrochloride and counterstained with hemalum-eosin.

\section{Western blot studies of mouse thyroid tissue}

Proteins prepared from mouse thyroid tissue collected in RIPA buffer and sonicated were quantified using the BCA protein assay (Thermo Fisher Scientific). Then, $20 \mu \mathrm{g}$ of total protein was separated on Bis-Tris polyacrylamide gel with a $4-12 \%$ gradient (Thermo Fisher Scientific) and transferred onto PVDF membranes (Thermo Fisher Scientific). The membranes were incubated with the primary antibodies mouse anti-Chop $(1: 1,000$, \# 2895, Cell Signaling Technology, Danvers, MA, USA) or rabbit anti-Actin $(1: 2,000$, \# A5441, Sigma-Aldrich) antibodies, followed by horseradish peroxidase-conjugated goat anti-mouse or anti-rabbit antibodies. Binding of secondary antibodies was revealed using the Amersham ECL Prime Detection Reagent Kit (GE Healthcare, Chicago, IL, USA). The protein bands on the membranes were scanned with the ImageQuant LAS 4000 Station (GE Healthcare) and then analysed using ImageJ $1.32 \mathrm{~s}$ to determine the protein levels, with Actin protein serving as an internal control.

\section{Molecular modelling of the P160L mutated protein}

The wild-type human TUBB1 sequence (accession number: Q9H4B7) was downloaded from the UniProt database, and the P160L mutation introduced into it. Both the wild-type and mutant TUBB1 sequences were modelled using Modeller 9.18 software (Šali \& Blundell, 1993) with PDB 4I4T chain B as the template (Prota et al, 2013). The models were analysed using PyMOL visualization software (DeLano, 2002).

\section{Electron microscopy}

Samples were fixed for $1 \mathrm{~h}$ in $3 \%$ glutaraldehyde in PBS buffer, washed and embedded in Epon. 90-nm sections were collected on nickel grids and contrasted with uranyl acetate and lead citrate. Acquisitions were performed with a Gatan Orius 1000 CCD Camera (Gatan, Pleasanton, CA, USA) on a JEOL 1011 transmission electron microscope (JEOL, Tokyo, Japan).

\section{Plasmids, cell cultures, transfection and immunofluorescence}

We used the phumanTUBB1-tagged Myc vector described by Kunishima et al (2009). Mutant P160L-TUBB1 was generated using a PCR-based site-directed mutagenesis method as described previously, using the Stratagene QuikchangeVR Kit (Agilent Technologies; Carré et al, 2007). Nthy (Nthy-ori 3.1; given by Corinne Dupuy) immortalized human thyroid-cell lines were cultured as previously described and used from passage 12 (Lemoine et al, 1989). The Nthy cells were plated at $0.4 \times 10^{5} /$ well on poly-L-lysine-coated slides in 12 -well plates $24 \mathrm{~h}$ before transfection then transfected with $500 \mathrm{ng}$ of vectors containing wild-type or P160L mutant TUBB1 using XtremeGENE-HP-DNA, as recommended by the manufacturer (Roche Applied Science, Penzberg, Germany). After 24 h, cells were used for immunofluorescence as already described (Bourg et al, 2015). The cells were washed with pre-warmed PHEM buffer; fixed with $4 \%$ PFA, $0.2 \%$ glutaraldehyde and $0.5 \%$ Triton; and permeabilized with PBS-Triton $0.1 \%$. Immunostaining was performed with rabbit antiMyc antibody (\# 2272, 1:500, Cell Signaling Technology) and mouse anti- $\alpha$-tubulin (DM1A, \# T9026, 1:1,000, Sigma-Aldrich, Saint-Louis, MI, USA) then with Alexa Fluor 647 goat anti-rabbit and Alexa Fluor 555 goat anti-mouse antibodies (1:400, Thermo Fisher Scientific).

\section{Human megakaryocytes and proplatelet formation}

$\mathrm{CD} 4^{+}$cells were isolated from peripheral blood using an immunomagnetic technique according to the manufacturer's instructions (\#130-046-70, Miltenyi Biotec, Bergisch Gladbach, Germany). Briefly, $100 \mu \mathrm{l} \mathrm{FcR} \mathrm{blocking} \mathrm{reagent} \mathrm{and} 100 \mu \mathrm{l}$ CD34 MicroBeads were incubated with $10^{8}$ cells. The remaining population was cultured at $37^{\circ} \mathrm{C}$ in $5 \% \mathrm{CO}_{2}$ in Iscove's modified Dulbecco's medium (IMDM; Thermo Fisher Scientific) supplemented with 15\% BIT 9500 serum substitute (Stemcell Technologies, Vancouver, Canada), $\alpha$ monothioglycerol (Sigma-Aldrich) and liposomes (phosphatidylcholine, cholesterol and oleic acid; all from Sigma-Aldrich), in the presence of human recombinant stem cell factor (SCF, $20 \mathrm{ng} / \mathrm{ml}$, Miltenyi Biotec) and human thrombopoeitin (50 nM, Miltenyi Biotec) added once on day 0 to the culture medium, followed by $20 \mathrm{nM}$ thrombopoeitin alone on day 6 with no further SCF addition. For proplatelet formation assays, megakaryocytes were plated on a BSA-coated surface (chamber slide, Ibidi, Martinsried, Germany) on day 10 . On day 13 or 14 , the megakaryocytes were fixed using $4 \%$ paraformaldehyde and stained for $\beta$-tubulin.

\section{Preparation of washed platelets}

To obtain human platelets, venous blood from healthy donors or patients was collected in 10\% ACD/A buffer (75 mM sodium citrate, $44 \mathrm{mM}$ citric acid, $136 \mathrm{mM}$ dextrose, $\mathrm{pH}$ 4.5). Platelets were washed as previously described (Adam et al, 2003) in the presence of apyrase $(100 \mathrm{mU} / \mathrm{ml})$ and prostaglandin $\mathrm{E} 1(1 \mu \mathrm{M})$ to minimize platelet activation. Platelet counts in patients and controls were adjusted to similar levels $\left(3 \times 10^{8}\right.$ platelets $\left./ \mathrm{ml}\right)$ in Tyrode's buffer (137 mM NaCl, $2 \mathrm{mM} \mathrm{KCl}, 0.3 \mathrm{mM} \mathrm{NaH} \mathrm{PO}_{4}, 1 \mathrm{mM} \mathrm{MgCl}$, $5.5 \mathrm{mM}$ glucose, $5 \mathrm{mM} \mathrm{N}$-2-hydroxyethylpiperazine- $\mathrm{N}^{\prime}$-2-ethanesulfonic acid, $12 \mathrm{mM} \mathrm{NaHCO}_{3}$ and $2 \mathrm{mM} \mathrm{CaCl}_{2}, \mathrm{pH}$ 7.3).

\section{Platelet aggregation}

Platelet aggregation was monitored by measuring light transmission through a stirred suspension of washed platelets $\left(3 \times 10^{8} / \mathrm{ml}\right)$ at $37^{\circ} \mathrm{C}$ using a Chrono-Log Aggregometer (Chrono-Log Corporation, Havertown, PA, USA), as previously described (Adam et al, 2010). Platelet aggregation was triggered by ADP and type I collagen (Chrono-log Corp.). 


\section{Flow cytometry of human platelets}

Whole blood from healthy donors or patients was diluted in PBS to obtain a platelet concentration of $2.5 \times 10^{7} / \mathrm{ml}$. Diluted whole blood was then incubated with phycoerythrin (PE)-anti-human CD62P (clone AK-4; eBioscience, Thermo Fisher Scientific, $5 \mu$ l anti-CD62P$\mathrm{PE} / 5 \times 10^{5}$ platelets) or fluorescein isothiocyanate (FITC) antihuman-activated $\alpha_{\mathrm{IIb}} \beta_{3}$ integrin (clone PAC-1; \#340507, Becton Dickinson, $20 \mu \mathrm{l} \mathrm{PAC-1-FITC/5} \times 10^{5}$ platelets) according to manufacturer's instructions for $20 \mathrm{~min}$ at room temperature. The samples were then analysed directly with an Accuri C6 flow cytometer (Becton Dickinson).

\section{Western blotting study of human platelets}

Washed platelets $\left(300 \mu \mathrm{l} ; 3 \times 10^{8} / \mathrm{ml}\right)$ were lysed in Laemmli sample buffer (10 mM HEPES, 2\% SDS, 10\% glycerol and $5 \mathrm{mM}$ EDTA). The proteins were separated by sodium dodecyl sulphate (SDS)-polyacrylamide gel electrophoresis and transferred to nitrocellulose membranes, which were incubated with the primary antibodies rabbit anti- $\alpha$ tubulin (1:1,000, Clone EP1332Y; Merck Millipore, Billerica, MA, USA) or rabbit anti- $\beta 1$-tubulin antibody $(1: 1,000$, donated by François Lanza). Immunoreactive bands were visualized with enhanced chemiluminescence detection reagents (Perbio Science, Thermo Fisher Scientific) using a G:BOX Chemi XT16 Image System and then quantified using Gene Tools version 4.03.05.0 (Syngene, Cambridge, UK).

\section{Statistics}

Sample size determination was based on previous experience with similar studies. Results are reported as mean \pm SEM for the number of experiments indicated in the figure legends. Statistical analyses were performed using GraphPad Prism4 (GraphPad, La Jolla, CA, USA). Data were analysed by one-way ANOVA followed by Dunnett's test, except for those parameters involving comparison of only two experimental groups, in which case an unpaired Student's $t$-test was used as indicated in the figure legends. Differences were considered significant when $P<0.05$. All $P$-values for figures can be found in Appendix Table S3.

\section{Data availability}

The datasets produced in this study are available in the following database: Clinical data: ClinVar accession numbers: SCV0008 40553.1, SCV000840554.1 and SCV000840555.1 (https://www.ncbi. nlm.nih.gov/clinvar/?term = SCV000840553.1, http://www.ncbi.nlm. nih.gov/clinvar/?term = SCV000840554.1 and http://www.ncbi.nlm. nih.gov/clinvar/?term = SCV000840555.1).

Expanded View for this article is available online.

\section{Acknowledgements}

This study was funded by the French public non-profit funding agency Programme Hospitalier de Recherche Clinique (ClinicalTrial.gov: NCT01916018, P 110120 - IDRCB 2012-A00797-36). AC, and MP received financial support from three corporations (EDF, Sandoz SAS, and Merck Serono France) and from the

\section{The paper explained}

Problem

Congenital hypothyroidism is the most common endocrine neonatal disorder with an estimated prevalence of $1 / 3,500$ newborns. It is mainly caused by defects in thyroid development (thyroid dysgenesis) or thyroid hormone synthesis. Among patients with congenital hypothyroidism due to thyroid dysgenesis, the specific genetic cause is identified only in $<5 \%$ of patients. Identifying the molecular defect allows early clinical care of hypothyroidism and associated malformations, and provides new insights into thyroid development and disease.

\section{Results}

Using whole exome sequencing, we uncovered an homozygous missense mutation in TUBBI gene, in two siblings of a consanguineous family with congenital hypothyroidism and thyroid dysgenesis (TD). By direct TUBB1 sequencing in a cohort of 270 patients with TD, we identified two more mutations in two distinct families with TD. TUBB1 gene encodes for a $\beta 1$-tubulin, and until now, reported roles for this protein were confined to platelets. Our functional studies show TUBB1 expression in the developing and adult thyroid in humans and mice. The Tubb1 $1^{-1-}$ mice have large platelets and show hypothyroidism, in accordance with the phenotype in mutated patients. Thyroids of Tubb1 ${ }^{-1-}$ exhibited proliferation defects during early development (embryonic day E9.5), altered migration at E11.5 and E13.5, and failure of hormone secretion at E17.5 and adulthood. All these mechanisms require proper microtubule function. Interestingly, two of the novel TUBBI mutations were associated with basal activation and exaggerated aggregation of platelets.

\section{Impact}

This is the first time that TUBBI mutations are associated with thyroid dysgenesis, in addition to abnormal platelet physiology. These findings expand the spectrum of the rare paediatric diseases related to tubulin mutations and provide new insights into the genetic background and mechanisms involved in congenital hypothyroidism. A TUBB1 mutation screening study in patients with non-autoimmune, non-postsurgical hypothyroidism and altered mean platelet volume and/or a history of thrombotic disease should be considered.

non-profit Princess Grace Foundation of Monaco. AC was funded by SFEDP (Société Française d’Endocrinologie et Diabétologie Pédiatrique). AS was supported by the European Society for Paediatric Endocrinology Research Fellowship Grant and Alexander S. Onassis Foundation. CJ has received support under the program "Investissements d'Avenir" launched by the French Government and implemented by ANR with the references ANR-10-LBX-0038, ANR10-IDEX-0001-02 PSL. KN was supported by the FRM fellowship SPF20140129173. We thank the patients and families for their participation, the URC-CIC Paris Centre (Sandra Colas and Emilie Ervilus) for study implementation and monitoring, and the IMAGINE Institute Biobank. We are deeply grateful to Beatrice Durel and Pierre Bourdoncle at the Cochin Imaging Facility. Our gratitude extends also to Monique Freund and her team at the animal facility in Strasbourg for technical assistance with the animal studies. We thank Prof. Samuel Refetoff (University of Chicago) for performing the plasma hormonal assays; and Daphné Geloen (Necker Children's Hospital, Biological Haematology Department, Paris) and Christelle Repérant (U1176 INSERM, Le Kremlin-Bicêtre) for their technical assistance with the platelet studies.

\section{Author contributions}

$A C$ and MP coordinated and instigated the study with FA and DB. ASt, DK, JL and MP provided clinical samples and data. AC, DK, ASt, CB, AMu and MP 
planned the genetic work. $A C$ and $C B$ performed exome sequencing data. $A C$ performed molecular studies and imaging data. CS and SG performed flow cytometry studies. FJ-H and FT performed Burden test. AC, FA, DB and MP analysed the data. CS and FL provided mice and performed mouse platelets studies. FA, AK, DL and DB performed human platelets studies. KN performed the modelling studies. CB-F coordinated the WES procedure. PN gave bioinformatics support. ASC and AMi performed electron microscopy studies. AC, ASt, FA and MP draft and finalized the manuscript with the help of DB, CJ, $\mathrm{FL}$, and RS.

\section{Conflict of interest}

The authors declare that they have no conflict of interest.

\section{For more information}

(i) ExAC Browser, http://exac.broadinstitute.org

(ii) 1000 Genomes, http://www.1000genomes.org

(iii) dbSNP, http://www.ncbi.n/m.nih.gov/projects/SNP/

(iv) PolyPhen-2, http://genetics.bwh.harvard.edu/pph2/

(v) SIFT, http://sift.bii.a-star.edu.sg

\section{References}

Adam F, Verbeuren TJ, Fauchère JL, Guillin MC, Jandrot-Perrus M (2003) Thrombin-induced platelet PAR4 activation: role of glycoprotein Ib and ADP. J Thromb Haemost 1: 798-804

Adam F, Kauskot A, Nurden P, Sulpice E, Hoylaerts MF, Davis RJ, Rosa JP, Bryckaert M (2010) Platelet JNK1 is involved in secretion and thrombus formation. Blood 115: 4083-4092

Amendola E, De Luca P, Macchia PE, Terracciano D, Rosica A, Chiappetta G, Kimura S, Mansouri A, Affuso A, Arra C et al (2005) A mouse model demonstrates a multigenic origin of congenital hypothyroidism. Endocrinology 146: 5038 - 5047

Barry Y, Bonaldi C, Goulet V, Coutant R, Léger J, Paty AC, Delmas D, Cheillan D, Roussey M (2016) Increased incidence of congenital hypothyroidism in France from 1982 to 2012: a nationwide multicenter analysis. Ann Epidemiol 26: $100-105$

Bastida JM, Lozano ML, Benito R, Janusz K, Palma-Barqueros V, Del Rey M, Hernández-Sánchez JM, Riesco S, Bermejo N, González-García H et al (2018) Introducing high-throughput sequencing into mainstream genetic diagnosis practice in inherited platelet disorders. Haematologica 103: $148-162$

Boehm U, Bouloux PM, Dattani MT, de Roux N, Dodé C, Dunkel L, Dwyer AA, Giacobini P, Hardelin JP, Juul A et al (2015) Expert consensus document: European Consensus Statement on congenital hypogonadotropic hypogonadism-pathogenesis, diagnosis and treatment. Nat Reu Endocrinol 11: $547-564$

Bonifacino JS, Neefjes J (2017) Moving and positioning the endolysosomal system. Curr Opin Cell Biol 47: 1-8

Bourg N, Mayet C, Dupuis C, Barroca T, Bon P, Lécart S, Fort E, Lévêque-Fort $S$ (2015) Direct optical nanoscopy with axially localized detection. Nat Photonics 9: 587-593

Breuss M, Heng JI, Poirier K, Tian G, Jaglin XH, Qu Z, Braun A, Gstrein T, Ngo L, Haas $M$ et al (2012) Mutations in the $\beta$-tubulin gene TUBB5 cause microcephaly with structural brain abnormalities. Cell Rep 2: 1554-1562

Burley K, Westbury SK, Mumford AD (2018) TUBB1 variants and human platelet traits. Platelets 29: 209-211

Carré A, Castanet M, Sura-Trueba S, Szinnai G, Van Vliet G, Trochet D, Amiel J, Léger J, Czernichow P, Scotet V et al (2007) Polymorphic length of FOXE1 alanine stretch: evidence for genetic susceptibility to thyroid dysgenesis. Hum Genet 122: 467-476

Carré A, Hamza RT, Kariyawasam D, Guillot L, Teissier R, Tron E, Castanet M, Dupuy C, El Kholy M, Polak M (2014) A novel FOXE1 mutation (R73S) in Bamforth-Lazarus syndrome causing increased thyroidal gene expression. Thyroid 24: 649-654

Carre A, Rachdi L, Tron E, Richard B, Castanet M, Schlumberger M, Bidart JM, Szinnai G, Polak M (2011) Hes1 is required for appropriate morphogenesis and differentiation during mouse thyroid gland development. PLoS One 6: e16752

Carré A, Szinnai G, Castanet M, Sura-Trueba S, Tron E, Broutin-L'Hermite I, Barat P, Goizet C, Lacombe D, Moutard ML et al (2009) Five new TTF1/ NKX2.1 mutations in brain-lung-thyroid syndrome: rescue by PAX8 synergism in one case. Hum Mol Genet 18: 2266-2276

Carré A, Stoupa A, Kariyawasam D, Gueriouz M, Ramond C, Monus T, Léger J, Gaujoux S, Sebag F, Glaser N et al (2017) Mutations in BOREALIN cause thyroid dysgenesis. Hom Mol Genet 26: 599-610

Carvalho DP, Dupuy C (2017) Thyroid hormone biosynthesis and release. Mol Cell Endocrinol 458: 6-15

Chakraborti S, Natarajan K, Curiel J, Janke C, Liu J (2016) The emerging role of the tubulin code: from the tubulin molecule to neuronal function and disease. Cytoskeleton (Hoboken) 73: 521-550

Choi M, Scholl UI, Ji W, Liu T, Tikhonova IR, Zumbo P, Nayir A, Bakkaloğlu A, Ozen S, Sanjad S et al (2009) Genetic diagnosis by whole exome capture and massively parallel DNA sequencing. Proc Natl Acad Sci USA 106: 19096-19101

Deladoëy J, Vassart G, Van Vliet G (2007) Possible non-Mendelian mechanisms of thyroid dysgenesis. Endocr Deu 10: 29-42

Deladoëy J, Ruel J, Giguère Y, Van Vliet G (2011) Is the incidence of congenita hypothyroidism really increasing? A 20-year retrospective populationbased study in Québec. J Clin Endocrinol Metab 96: 2422-2429

DeLano WL (2002) The PyMOL molecular graphics system.

Dentice M, Cordeddu V, Rosica A, Ferrara AM, Santarpia L, Salvatore D, Chiovato L, Perri A, Moschini L, Fazzini C et al (2006) Missense mutation in the transcription factor NKX2-5: a novel molecular event in the pathogenesis of thyroid dysgenesis. J Clin Endocrinol Metab 91: $1428-1433$

Di Jeso B, Arvan P (2016) Thyroglobulin from molecular and cellular biology to clinical endocrinology. Endocr Reu 37: 2-36

de Filippis T, Marelli F, Nebbia G, Porazzi P, Corbetta S, Fugazzola L, Gastaldi R, Vigone MC, Biffanti R, Frizziero D et al (2016) JAG1 loss-of-function variations as a novel predisposing event in the pathogenesis of congenital thyroid defects. J Clin Endocrinol Metab 101: 861-870

de Filippis T, Gelmini G, Paraboschi E, Vigone MC, Di Frenna M, Marelli F, Bonomi M, Cassio A, Larizza D, Moro M et al (2017) A frequent oligogenic involvement in congenital hypothyroidism. Hum Mol Cenet 26: 2507-2514

Fiore M, Goulas C, Pillois X (2017) A new mutation in TUBB1 associated with thrombocytopenia confirms that $\mathrm{C}$-terminal part of $\beta 1$-tubulin plays a role in microtubule assembly. Clin Genet 91: 924-926

Franchini M, Lippi G, Manzato F, Vescovi PP, Targher G (2010) Hemostatic abnormalities in endocrine and metabolic disorders. Eur J Endocrinol 162: 439-451

Gaide Chevronnay HP, Janssens V, Van Der Smissen P, Liao XH, Abid Y, Nevo N, Antignac C, Refetoff S, Cherqui S, Pierreux CE et al (2015) A mouse model suggests two mechanisms for thyroid alterations in infantile cystinosis: decreased thyroglobulin synthesis due to endoplasmic reticulum stress/unfolded protein response and impaired lysosomal processing. Endocrinology 156: 2349-2364 
Gawade S, Mayer C, Hafen K, Barthlott T, Krenger W, Szinnai G (2016) Cell growth dynamics in embryonic and adult mouse thyroid revealed by a novel approach to detect thyroid gland subpopulations. Thyroid 26: $591-599$

Gordon CT, Petit F, Kroisel PM, Jakobsen L, Zechi-Ceide RM, Oufadem M, Bole-Feysot C, Pruvost S, Masson C, Tores F et al (2013) Mutations in endothelin 1 cause recessive auriculocondylar syndrome and dominant isolated question-mark ears. Am J Hum Genet 93: 1118-1125

Guillot L, Carré A, Szinnai G, Castanet M, Tron E, Jaubert F, Broutin I, Counil F, Feldmann D, Clement A et al (2010) NKX2-1 mutations leading to surfactant protein promoter dysregulation cause interstitial lung disease in 'Brain-Lung-Thyroid Syndrome'. Hum Mutat 31: E1146-E1162

Hildebrandt F, Heeringa SF, Rüschendorf F, Attanasio M, Nürnberg G, Becker C, Seelow D, Huebner N, Chernin G, Vlangos CN et al (2009) A systematic approach to mapping recessive disease genes in individuals from outbred populations. PLoS Genet 5: e1000353

Huotari J, Helenius A (2011) Endosome maturation. EMBO J 30: $3481-3500$

Janke C (2014) The tubulin code: molecular components, readout mechanisms, and functions. J Cell Biol 206: 461-472

Joe PA, Banerjee A, Ludueña RF (2009) Roles of beta-tubulin residues Ala428 and Thr429 in microtubule formation in vivo. J Biol Chem 284: 4283-4291

Johnson B, Lowe GC, Futterer J, Lordkipanidzé M, MacDonald D, Simpson MA, Sanchez-Guiú I, Drake S, Bem D, Leo V et al, (2016) Whole exome sequencing identifies genetic variants in inherited thrombocytopenia with secondary qualitative function defects. Haematologica 101: 1170-1179

Kariyawasam D, Rachdi L, Carré A, Martin M, Houlier M, Janel N, Delabar JM, Scharfmann R, Polak M (2015) DYRK1A BAC transgenic mouse: a new model of thyroid dysgenesis in Down syndrome. Endocrinology 156: 171-180

Kim JH, Park JH, Kim SY, Bae HY (2013) The mean platelet volume is positively correlated with serum thyrotropin concentrations in a population of healthy subjects and subjects with unsuspected subclinical hypothyroidism. Thyroid 23: 31-37

Kimura S, Hara Y, Pineau T, Fernandez-Salguero P, Fox CH, Ward JM, Gonzalez FJ (1996) The T/ebp null mouse: thyroid-specific enhancerbinding protein is essential for the organogenesis of the thyroid, lung, ventral forebrain, and pituitary. Cenes Deu 10: 60-69

Kircher M, Witten DM, Jain P, O'Roak BJ, Cooper GM, Shendure J (2014) A general framework for estimating the relative pathogenicity of human genetic variants. Nat Genet 46: 310-315

Kuhli-Hattenbach C, Hellstern P, Kohnen T, Hattenbach LO (2017) Platelet activation by ADP is increased in selected patients with anterior ischemic optic neuropathy or retinal vein occlusion. Platelets 28 : $720-723$

Kunishima S, Kobayashi R, Itoh TJ, Hamaguchi M, Saito H (2009) Mutation of the beta-1-tubulin gene associated with congenital macrothrombocytopenia affecting microtubule assembly. Blood 113: $458-461$

Kunishima S, Nishimura S, Suzuki H, Imaizumi M, Saito H (2014) TUBB1 mutation disrupting microtubule assembly impairs proplatelet formation and results in congenital macrothrombocytopenia. Eur J Haematol 92: $276-282$

Kyriakakis N, Lynch J, Ajjan R, Murray RD (2016) The effects of pituitary and thyroid disorders on haemostasis: potential clinical implications. Clin Endocrinol (Oxf) 84: 473-484

Ladoux B, Mège RM, Trepat X (2016) Front-rear polarization by mechanical cues: from single cells to tissues. Trends Cell Biol 26: $420-433$

Lecine P, Italiano JE Jr, Kim SW, Villeval JL, Shivdasani RA (2000) Hematopoieticspecific beta 1 tubulin participates in a pathway of platelet biogenesis dependent on the transcription factor NF-E2. Blood 96: 1366-1373
Lemoine NR, Mayall ES, Jones T, Sheer D, McDermid S, Kendall-Taylor P, Wynford-Thomas D (1989) Characterisation of human thyroid epithelial cells immortalised in vitro by simian virus 40 DNA transfection. $\mathrm{Br}$ J Cancer 60: 897-903

Magne F, Ge B, Larrivée-Vanier S, Van Vliet G, Samuels ME, Pastinen T, Deladoëy J (2016) Demonstration of autosomal monoallelic expression in thyroid tissue assessed by whole-exome and bulk RNA sequencing. Thyroid 26: $852-859$

Maiorana R, Carta A, Floriddia G, Leonardi D, Buscema M, Sava L, Calaciura F, Vigneri R (2003) Thyroid hemiagenesis: prevalence in normal children and effect on thyroid function. J Clin Endocrinol Metab 88: $1534-1536$

Mansouri A, Chowdhury K, Gruss P (1998) Follicular cells of the thyroid gland require Pax8 gene function. Nat Genet 19: 87-90

Morgenthaler S, Thilly WG (2007) A strategy to discover genes that carry multi-allelic or mono-allelic risk for common diseases: a cohort allelic sums test (CAST). Mutat Res 615: $28-56$

Muller J, Stoetzel C, Vincent MC, Leitch CC, Laurier V, Danse JM, Hellé S, Marion V, Bennouna-Greene V, Vicaire S et al (2010) Identification of 28 novel mutations in the Bardet-Biedl syndrome genes: the burden of private mutations in an extensively heterogeneous disease. Hum Genet 127: $583-593$

Nève P, Ketelbant-Balasse P, Willems C, Dumont JE (1972) Effect of inhibitors of microtubules and microfilaments on dog thyroid slices in vitro. Exp Cell Res 74: $227-244$

Nilsson M, Fagman H (2013) Mechanisms of thyroid development and dysgenesis: an analysis based on developmental stages and concurrent embryonic anatomy. Curr Top Dev Biol 106: 123-170

Nilsson M, Fagman H (2017) Development of the thyroid gland. Development 144: $2123-2140$

Nirschl JJ, Magiera MM, Lazarus JE, Janke C, Holzbaur EL (2016) $\alpha$-Tubulin tyrosination and CLIP-170 phosphorylation regulate the initiation of dynein-driven transport in neurons. Cell Rep 14: 2637-2652

Nogales E, Wolf SC, Downing KH (1998) Structure of the alpha beta tubulin dimer by electron crystallography. Nature 391: 199-203

Opitz R, Hitz MP, Vandernoot I, Trubiroha A, Abu-Khudir R, Samuels M, Désilets V, Costagliola S, Andelfinger G, Deladoëy J (2015) Functional zebrafish studies based on human genotyping point to netrin-1 as a link between aberrant cardiovascular development and thyroid dysgenesis. Endocrinology 156: $377-388$

Patel SR, Richardson JL, Schulze H, Kahle E, Galjart N, Drabek K, Shivdasani RA, Hartwig JH, Italiano JE Jr (2015) Differential roles of microtubule assembly and sliding in proplatelet formation by megakaryocytes. Blood 106: $4076-4085$

Pohlenz J, Maqueem A, Cua K, Weiss RE, Van Sande J, Refetoff S (1999) Improved radioimmunoassay for measurement of mouse thyrotropin in serum: strain differences in thyrotropin concentration and thyrotroph sensitivity to thyroid hormone. Thyroid 9: $1265-1271$

Prosser SL, Pelletier L (2017) Mitotic spindle assembly in animal cells: a fine balancing act. Nat Reu Mol Cell Biol 18: 187-201

Prota AE, Bargsten K, Zurwerra D, Field JJ, Díaz JF, Altmann KH, Steinmetz MO (2013) Molecular mechanism of action of microtubule-stabilizing anticancer agents. Science 339: 587-590

Raiborg C, Wenzel EM, Stenmark H (2015) ER-endosome contact sites: molecular compositions and functions. EMBO J 34: 1848-1858

Ramos HE, Carré A, Chevrier L, Szinnai G, Tron E, Cerqueira TL, Léger J, Cabrol S, Puel O, Queinnec C et al (2014) Extreme phenotypic 
variability of thyroid dysgenesis in six new cases of congenital hypothyroidism due to PAX8 gene loss-of-function mutations. Eur J Endocrinol 171: 499-507

Rousset B, Dupuy C, Miot F, Dumont J (2015) Chapter 2 thyroid hormone synthesis and secretion. In Endotext, De Groot LJ, Chrousos G, Dungan K, Feingold KR, Grossman A, Hershman JM, Koch C, Korbonits M, McLachlan R, New M, Purnell J, Rebar R, Singer F, Vinik A (eds), pp 2000-2015. South Dartmouth, MA: MDText.com Inc.

Šali A, Blundell TL (1993) Comparative protein modelling by satisfaction of spatial restraints. J Mol Biol 234: 779-815

Schwer HD, Lecine P, Tiwari S, Italiano JE Jr, Hartwig JH, Shivdasani RA (2001) A lineage-restricted and divergent beta-tubulin isoform is essential for the biogenesis, structure and function of blood platelets. Curr Biol 11: $579-586$

Senée V, Chelala C, Duchatelet S, Feng D, Blanc H, Cossec JC, Charon C, Nicolino M, Boileau P, Cavener DR et al (2006) Mutations in GLIS3 are responsible for a rare syndrome with neonatal diabetes mellitus and congenital hypothyroidism. Nat Genet 38: 682-687

Squizzato A, Romualdi E, Büller HR, Gerdes VE (2007) Clinical review: thyroid dysfunction and effects on coagulation and fibrinolysis: a systematic review. J Clin Endocrinol Metab 92: 2415-2420

Stoupa A, Kariyawasam D, Carré A, Polak M (2016) Update of thyroid developmental genes. Endocrinol Metab Clin North Am 45: 243-254

Sura-Trueba S, Aumas C, Carre A, Durif S, Leger J, Polak M, de Roux N (2009) An inactivating mutation within the first extracellular loop of the thyrotropin receptor impedes normal posttranslational maturation of the extracellular domain. Endocrinology 150: 1043-1050

Szinnai G, Lacroix L, Carré A, Guimiot F, Talbot M, Martinovic J, Delezoide AL, Vekemans M, Michiels S, Caillou B et al (2007) Sodium/iodide symporter (NIS) gene expression is the limiting step for the onset of thyroid function in the human fetus. J Clin Endocrinol Metab 92: 70-76

The 1000 Genomes Project Consortium (2015) A global reference for human genetic variation. Nature 526: $68-74$

Trueba SS, Augé J, Mattei G, Etchevers H, Martinovic J, Czernichow P, Vekemans M, Polak M, Attié-Bitach T (2005) PAX8, TITF1, and FOXE1 gene expression patterns during human development: new insights into human thyroid development and thyroid dysgenesis-associated malformations. J Clin Endocrinol Metab 90: 455-462

Wang D, Villasante A, Lewis SA, Cowan NJ (1986) The mammalian betatubulin repertoire: hematopoietic expression of a novel, heterologous beta-tubulin isotype. J Cell Biol 103: 1903-1910

Wildi-Runge S, Stoppa-Vaucher S, Lambert R, Turpin S, Van Vliet G, Deladoey J (2012) A high prevalence of dual thyroid ectopy in congenital hypothyroidism: evidence for insufficient signaling gradients during embryonic thyroid migration or for the polyclonal nature of the thyroid gland? J Clin Endocrinol Metab 97: E978-E981

Wolff J, Bhattacharyya B (1975) Microtubules and thyroid hormone mobilization. Ann N Y Acad Sci 253: 763-770

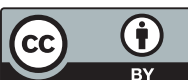

License: This is an open access article under the terms of the Creative Commons Attribution 4.0 License, which permits use, distribution and reproduction in any medium, provided the original work is properly cited. 